\title{
Presenteeism and Productivity Loss Among Employees of Iranian Gas Transmission Company Manufacturers
}

\author{
Jafar Akbari ${ }^{\text {* }}$ (D), Mahmood Abolghasemian', Amir Ghaffari², Fereydun Moghaddas ${ }^{1}$, \\ Mohammad Amin Mououdi ${ }^{3}$, Shaghayegh Rahimi Kamal ${ }^{4}$, Masoumeh JozKanani
}

1. Health, Safety, Environment (HSE) and Passive Defense, Isfahan Province Gas Company, National Iranian Gas Company, Isfahan, Iran

2. A Health, Safety, Environment (HSE) and Passive Defense, National Iranian Gas Company, Tehran, Iran

3. Department of Occupational Health Engineering, School of Public Health, Mazandaran University of Medical Sciences, Sari, Iran

4. Health, Safety, Environment (HSE) and Passive Defense, Iranian Gas Transmission Company, National Iranian Gas Company, Tehran, Iran

\section{Article Info \\ Received: 2021/01/27; \\ Accepted: 2021/07/25; \\ ePublished: 2021/09/07 \\ 10.30699/jergon.9.1.33 \\ Use your device to scan and read the article online

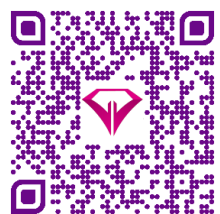

Corresponding Author Jafar Akbari

Health, Safety, Environment (HSE) and Passive Defense, Isfahan Province Gas Company, National Iranian Gas Company, Isfahan, Iran

Email:

akbari.jafar@gmail.com

\section{ABSTRACT}

Background and Objectives: Presenteeism is introduced as a phenomenon according to which employees are physically present in the workplace but are functionally absent. This study aimed to investigate presenteeism and productivity loss associated with health problems among employees of the Iranian Gas Transmission Company (IGTC).

Methods: The participants were 2298 employees. Data collection instruments included the Stanford Presenteeism Scale (SPS-6) and the Health and Work Questionnaire (HWQ). Data analysis was performed using the multivariate logistic regression by SPSS software version 26.

Results: More than $52 \%$ of the participants (1189 people) had experienced presenteeism at least once a year, while a total of 1073 people $(47 \%)$ were nonpresenteists. The results showed that the HWQ subsets including productivity $(P=0.001)$, focus $(P=0.023)$, relationships with the manager or boss $(P=0.007)$, impatience and irritability $(P=0.026)$, workplace satisfaction $(P=0.038)$ and finally the total HWQ score $(P=0.011)$ had a statistically significant relationship with presenteeism.

Conclusion: Presenteeism reduces the productivity of the workforce reducing individual productivity, reducing people's ability to concentrate, the inappropriate perception of the relationship with the manager/ boss, increasing their impatience, irritability and level of work-related stress and eventually reducing the level of people's satisfaction with the work environment. This imposes a lot of costs on the organization.

Keywords: Presenteeism, Productivity loss, Stanford Presenteeism Scale, Health and work, Employees of Iranian Gas Transmission Company

\section{How to Cite This Article:}

Akbari J, Abolghasemian M, Ghaffari A, Moghaddas F, Mououdi M A, Rahimi Kamal S et al. Presenteeism and productivity loss among employees of Iranian Gas Transmission Company. Iran J Ergon. 2021; 9 (1) :33-47 


\section{Extended Abstract}

\section{Introduction}

A global phenomenon, presenteeism is defined as the problem of workers who are on the job, however, because of illness or other medical conditions, not fully functioning [4]. The main concern of the phenomenon of presenteeism is mainly related to the economic impact of the loss of productivity of people who are present at work despite illness or discomfort [5]. In recent years, the study of the researches related to presenteeism has become popular among several researchers in the field of health, occupational psychology and occupational epidemiologists all around the world.

Although the importance of this phenomenon is completely known in the United States [14], Canada [11], Britain [15], the Netherlands [16], Denmark [17], Sweden [18], Taiwan [19], South Korea [20], Saudi Arabia Saudi Arabia [21] and other countries, unfortunately, in Iran no study has been conducted regarding the quality and quantity of presenteeism and its consequences among occupational groups.

The reason that studying the presenteeism and its adverse consequences is considered as an important phenomenon is that the presence of the presenteists in the organization can reduce their productivity level more than when they are absent; so, effective management of this phenomenon can help maintain and promote individual and organizational health. Therefore, the present study was conducted to investigate presenteeism and productivity loss associated with health problems among employees of the Iranian Gas Transmission Company (IGTC).

\section{Methods}

This cross-sectional study was performed among different job groups of the Iranian Gas Transmission Company (IGTC). After eliminating the incomplete questionnaires, the participants were 2298 employees (with a response rate of 96\%) including about $10 \%$ of supervisors or managers and $90 \%$ of worker and experts. Data was collected via demographic questionnaire as well as the two systematic instruments including the Stanford Presenteeism Scale (SPS-6) and the Health and Work Questionnaire (HWQ). All study data were organized in a spreadsheet program and analyzed by the statistical software. Descriptive statistics were used to obtain the frequency, frequency percentage and standard deviation (SD). Additionally, multivariate logistic regression test was utilized to investigate the relationship between presenteeism and productivity loss related to health problems. Accordingly, $95 \%$ odds ratio (OR) and 95\% confidence intervals $(95 \% \mathrm{CI})$ were reported for the study variables. Probability $(\mathrm{P})$ values below 0.05 were demonstrated as statistically significant throughout the analysis. The collected data were analyzed using SPSS software version 26 (SPSS Inc., Chicago, IL., USA).

This study has been approved by the ethics committee in the research of the Faculty of Health and Neuroscience of Shahid Beheshti University of Medical Sciences in Tehran with the ethics ID IR.SBMU.PHNS.REC.1397.092.

The data obtained in this study were processed with SPSS software version 24 (SPSS Inc., Chicago, Ill., USA). Data analysis was performed by Wilcoxon, paired t-test and sign test at a significance level of 0.05 .

\section{Results}

The majority of the participants were married (91.1\%) males (93.7\%), with an average age of 39 years. Of the subjects, 58.9 percent were well educated (bachelor, master and doctorate graduation). The participants had an average of 13.7 $( \pm 7.4)$ years of job tenure (range 1-30 years). The complete demographic profile of the participants is presented in Table 1 .

More than $52 \%$ of the participants (1189 people) had experienced presenteeism at least once a year, while a total of 1073 people (47\%) were nonpresenteists. The data also demonstrated that the mean score and standard deviation of Present- 
eeism were 20 and 4.7 , respectively. Figure 1 shows the average score of presenteeism based on the Stanford Presenteeism Scale (SPS-6) among employees of the Iranian Gas Transmission Company (IGTC).

\begin{tabular}{|c|c|c|c|c|}
\hline \multicolumn{2}{|c|}{ Variable } & $\begin{array}{c}\text { Mean }( \pm \mathbf{S D}) / \\
\text { frequency }\end{array}$ & Frequency percentage & $P^{*}$ \\
\hline \multicolumn{2}{|c|}{ Age (year) } & $39.91 \pm 9.67$ & - & 0.119 \\
\hline \multirow{2}{*}{ Sex } & Male & 2120 & 93.7 & \multirow{2}{*}{0.104} \\
\hline & Female & 143 & 6.3 & \\
\hline \multirow{2}{*}{ Marital status } & Single & 201 & 8.9 & \multirow{2}{*}{0.914} \\
\hline & Married & 2059 & 91.1 & \\
\hline \multirow{2}{*}{ Physical status } & Height (m) & $1.72 \pm 0.24$ & - & 0.042 \\
\hline & Weight (kg) & $80.70 \pm 12.89$ & - & 0.000 \\
\hline \multirow{5}{*}{$\begin{array}{c}\text { Body Mass Index } \\
\text { (BMI) }\end{array}$} & Weight loss & 18 & 08 & \multirow{5}{*}{0.000} \\
\hline & Natural & 799 & 35.4 & \\
\hline & Overweight & 1153 & 51.1 & \\
\hline & Obese & 248 & 11.0 & \\
\hline & Very obese & 39 & 1.7 & \\
\hline \multirow{5}{*}{ Education } & $\begin{array}{c}\text { Diploma and } \\
\text { lower }\end{array}$ & 562 & 24.9 & \multirow{5}{*}{0.166} \\
\hline & $\begin{array}{l}\text { Associate } \\
\text { Degree }\end{array}$ & 365 & 16.2 & \\
\hline & Bachelor & 705 & 31.2 & \\
\hline & MA & 608 & 26.9 & \\
\hline & Doctorate & 19 & 0.8 & \\
\hline \multirow{4}{*}{ Major } & Science & 447 & 19.9 & \multirow{4}{*}{0.010} \\
\hline & Engineering & 1237 & 55.0 & \\
\hline & Humanities & 546 & 24.3 & \\
\hline & $\begin{array}{l}\text { Medical \& } \\
\text { Health }\end{array}$ & 19 & 0.8 & \\
\hline \multirow{4}{*}{ Job } & Employee & 1550 & 68.5 & \multirow{4}{*}{0.742} \\
\hline & Expert & 476 & 21.0 & \\
\hline & Supervisor & 209 & 9.2 & \\
\hline & Manager & 27 & 1.2 & \\
\hline \multirow{3}{*}{ Job location } & Official & 584 & 25.8 & \multirow{3}{*}{0.337} \\
\hline & Central office & 436 & 19.3 & \\
\hline & Operational & 1243 & 54.9 & \\
\hline \multirow{3}{*}{$\begin{array}{l}\text { The type of } \\
\text { employment }\end{array}$} & Permanent & 784 & 34.6 & \multirow{3}{*}{0.135} \\
\hline & $\begin{array}{l}\text { temporary-to } \\
\text { permanent }\end{array}$ & 356 & 15.7 & \\
\hline & Contractual & 1123 & 49.6 & \\
\hline \multicolumn{2}{|c|}{ Job tenure (year) } & $13.71 \pm 7.47$ & - & 0.001 \\
\hline \multicolumn{2}{|c|}{ Working hours per week (hrs) } & $40 \pm 14.71$ & - & 0.000 \\
\hline \multicolumn{2}{|c|}{ Overtime per week (hrs) } & $12.15 \pm 10.33$ & - & 0.000 \\
\hline
\end{tabular}

*P-values below 0.05 were demonstrated as statistically significant throughout the analysis

**Weight loss: less than 18.5; Natural: 18.5-24.9; Overweight: 25-29.9; Obese: 30-34.9; Very obese: more than 35 (26). 


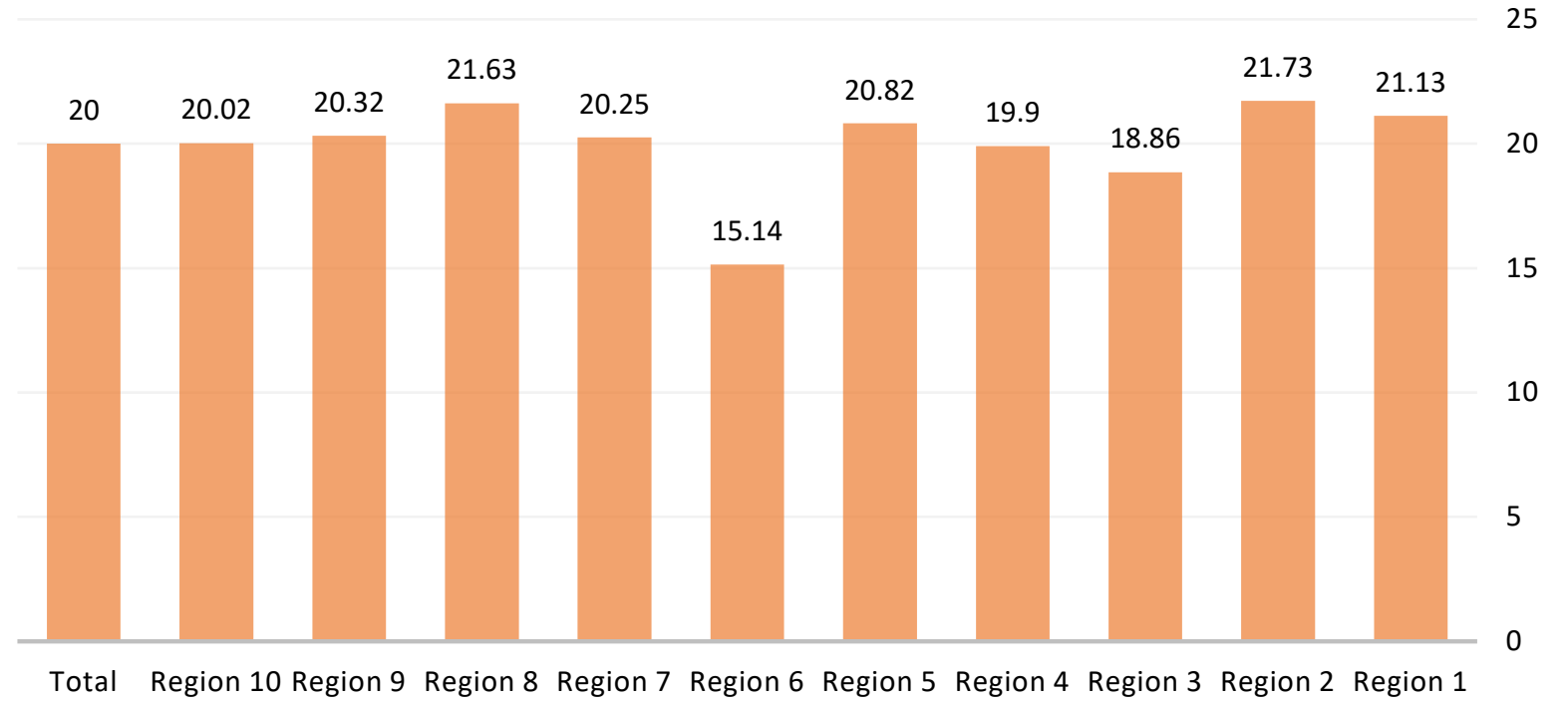

Figure 1. The average score of Presenteeism among the employees of the Iranian Gas Transmission Company (IGTC) (the final score of Presentism is between 6 and 30, the cut-off points of which is considered to be 18 . More than 18 are considered nonpresent).

The results of HWQ showed that the mean of overall score was clearly higher among presenteists than nonpresenteists. It is also revealed that in the four subgroups including productivity loss, relat-ionships with the boss or manager, job satisfaction and satisfaction outside the workplace, the present-eists reported higher mean scores than their count-erparts. Furthermore, the HWQ subsets including productivity ( $P=0.001)$, focus $(P=0.023)$, relation-ships with the manager or boss $(P=0.007)$, impa-tience and irritability $\quad(P=0.026)$, workplace satis-faction $(P=0.038)$ and finally the total HWQ score $(P=0.011)$ had a statistically significant relation-ship with presenteeism. Table 2 as well as Figure 2 show the average score of the Health and Work Questionnaire (HWQ) among the staff of the Iranian Gas Transmission Company (IGTC).

Table 2. Mean $( \pm \mathrm{SD})$ of the HWQ and its subcategories among the employees of the Iranian Gas Transmission Company (IGTC)

\begin{tabular}{|cccccccc|}
\hline Subcategories & \multicolumn{2}{c}{ Presenteists } & \multicolumn{2}{c}{ Non-presenteists } & \multicolumn{2}{c|}{ Total participants } & \multirow{2}{*}{$\boldsymbol{P}^{*}$} \\
& Mean & SD $^{* *}$ & Mean & SD & Mean & SD & \\
\hline Efficiency & 80.84 & 24.33 & 77.1 & 23.66 & 79.06 & 24.1 & 0.001 \\
\hline $\begin{array}{c}\text { Focus } \\
\text { Relationships with the } \\
\text { manager / boss }\end{array}$ & 15.57 & 5.61 & 16.22 & 4.95 & 15.88 & 5.31 & 0.023 \\
\hline $\begin{array}{c}\text { Impatience and irritability } \\
\text { Job satisfaction }\end{array}$ & 16.70 & 4.15 & 16.26 & 4.00 & 16.49 & 4.1 & 0.007 \\
\hline $\begin{array}{c}\text { Satisfaction outside the } \\
\text { workplace }\end{array}$ & 25.1 & 7.96 & 13.68 & 7.42 & 12.84 & 7.75 & 0.026 \\
\hline HWQ & 184.4 & 31.03 & 180.56 & 29.76 & 179.71 & 37.8 & 0.011 \\
\hline
\end{tabular}

*Probability (P) values below 0.05 were demonstrated as statistically significant throughout the analysis ** Standard Deviation 


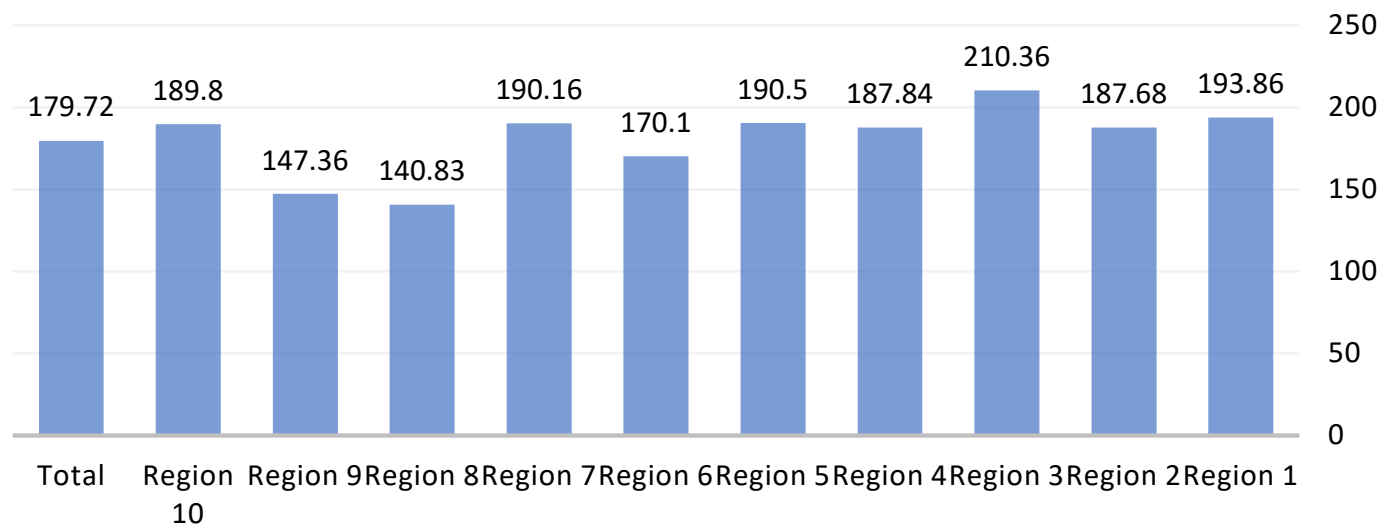

Figure 2. Comparison of the average score of the Health and Work Questionnaire (WHQ) among the employees of the Iranian Gas Transmission Company (IGTC) (this questionnaire has 24 questions with a total score of 300).

\section{Discussion}

This study firstly investigated the prevalence of presenteeism as an adverse global phenomenon and the associated factors among different job groups of the Iranian Gas Transmission Company (IGTC). In addition, we examined the effects of having presenteeism at least one time in the year on productivity loss related to the certain health condition. In this study, it was found that employees who had a higher level of body mass index (BMI) had mutually higher level of presenteeism rates and consequently lower work productivity. Regarding our findings, Saptavani et al. assessed the association between the worker's BMI and the reduction of productivity, presenteeism and absent-eeism. They reported that the body mass index is highly correlated with presenteeism and also workers' productivity. As a result, it is expected that productivity decreases will affect the indirect costs incurred by the company [28]. Also, Bustillos et al. surveyed work productivity and the prevalence of presenteeism among adults with varied BMI using population-based data. They found obesity as an independent risk factor for reduced work productivity. Also, they reported that both absenteeism and presenteeism were associated with obes-ity [29]. Other studies have also reported the effect of productivity loss in the workplace and the prevalence of presenteeism due to obesity and having a high BMI level [30].

The results of our study unraveled that presenteeism among employees reduces their job productivity in relation to their health condition. The prevalence of presenteeism among employees led to productivity loss by reducing individual productivity, people's ability to concentrate, the appropriate perception of the relationship with the manager/ boss, and by increasing the workers' impatience, irritability and level of work-related stress. Eventually, it was also occurred by reducing the level of people's satisfaction in relation with their work environment.

\section{Conclusion}

Presenteeism reduces the productivity of the workforce reducing individual productivity, reducing people's ability to concentrate, the inappropriate perception of the relationship with the manager/ boss, increasing their impatience, irritability and level of work-related stress and eventually reducing the level of people's satisfaction with the work environment. This imposes a lot of costs on the organization.

\section{Acknowledgement}

This study is the result of a comprehensive research implemented at the National Iranian Gas Company (NIGC). The authors would like to thank the employees of Industrial Hygiene and HSE section at Iran Gas Transmission Company who actively participated during data collection.

\section{Conflict of Interest}

The authors declared no conflict of interest. 


\title{
مطالعة يديدهُ يرزنتيسم و كاهش بهرهورى در بين كاركنان شركت انتقال كاز ايران
}

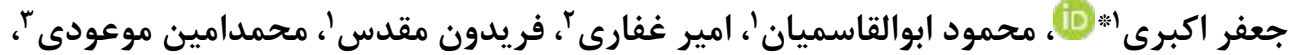

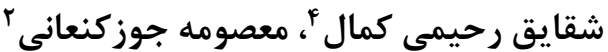

\begin{abstract}
l' بهداشت، ايمنى، محيط زيست (HSE) و پرافند غيرعامل، شركت كاز استان اصفهان، شركت ملى كاز ايران، اصفهان، ايران

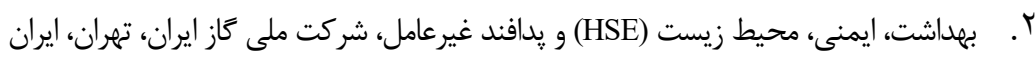

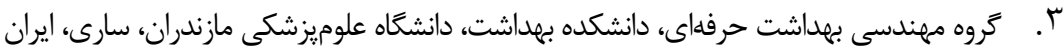

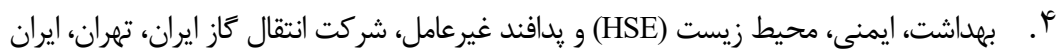

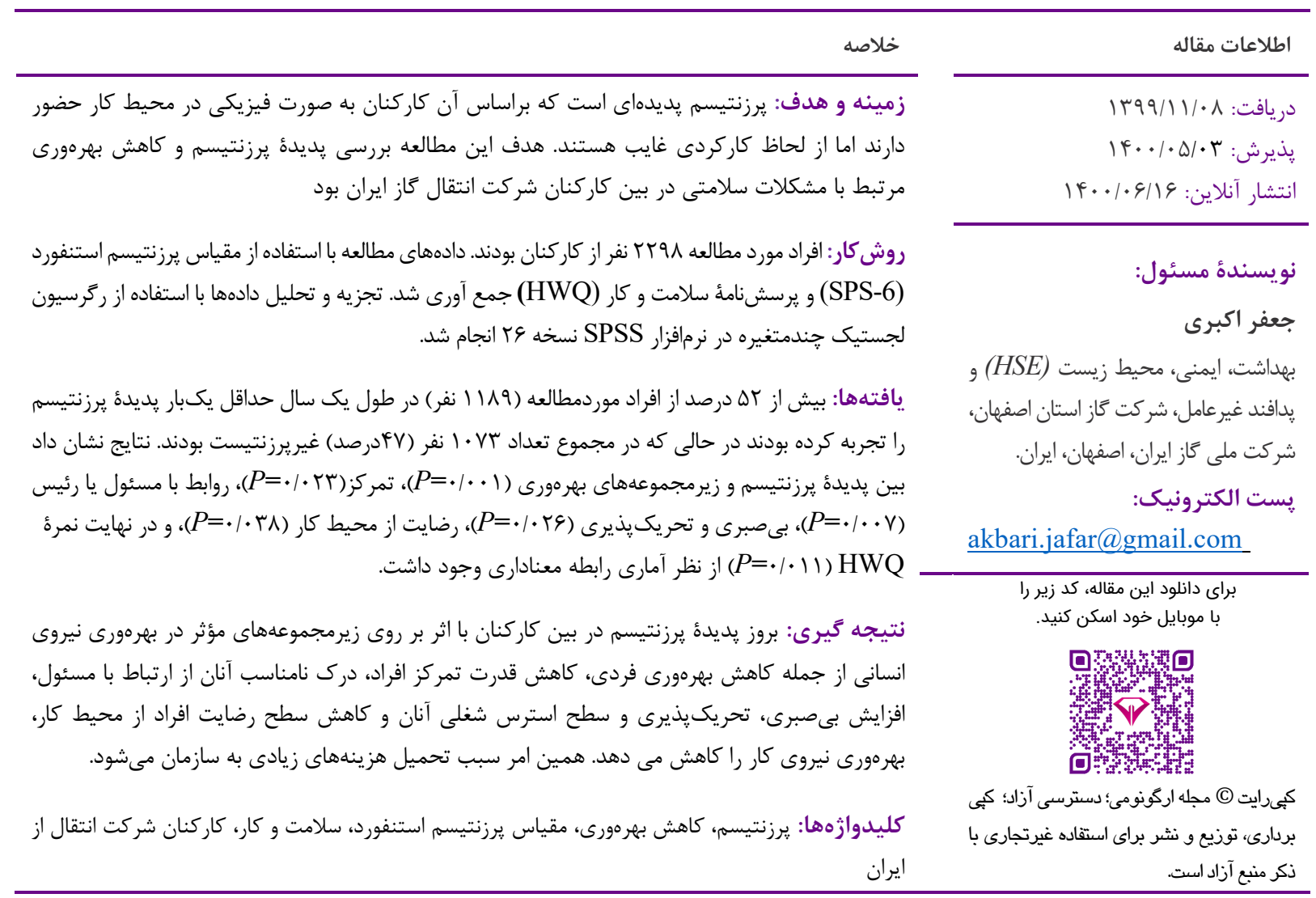

مقدمه

به مفهوم يرزنتيسمَّ، كه اغلب در گذشته به عنوان همتاى غيبت

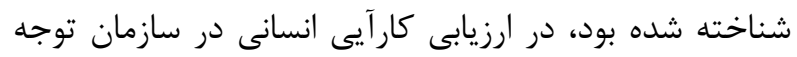

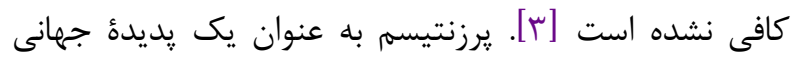
تحت عنوان مشكلات كارگرانى تعريف مى شود كه در كار حضور دارند اما به دليل بيمارى، كسالت، ناخوشى و يا ساير شرايط

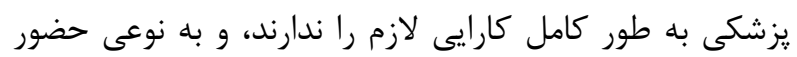

${ }^{2}$ Presenteeism
در دهdهاى كذشته، غيبت ' كه به عنوان يديده عدم حضور كاركنان براى انجام وظايف محوله و كارهاى برنامه ريزى شده تعريف مى شود به دليل تاثير آن بر بهرهورى سازمانهان بهان به طور

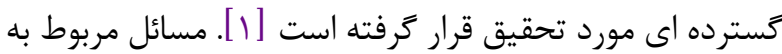
سلامتى به عنوان اصلى ترين دليل غيبت معرفى شده و أ درصد مدرد ضرر و زيان كاهش بهرهورى را تشكيل داده است [ب] بـ در مقابل،

${ }^{1}$ Absenteeism 
درمانى است، در حالى كه در ساير موارد (مانند فشارخون يا

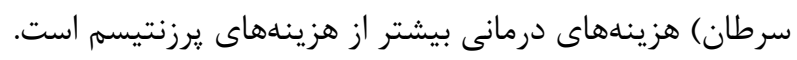

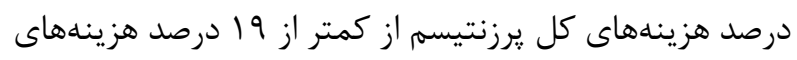

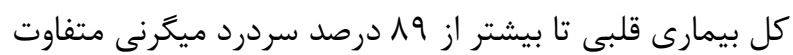

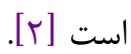

در سالهاى اخير، مطالعه بر روى بديده يرزنتيسم در بين

يزوهشكران حوزه سلامت، روانشناسى شغلى و إيدميولوزيست بردي

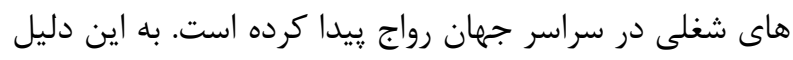

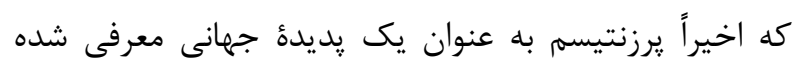

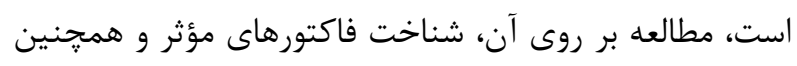

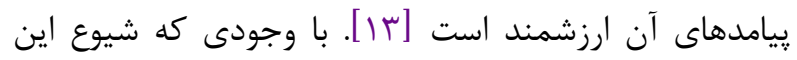

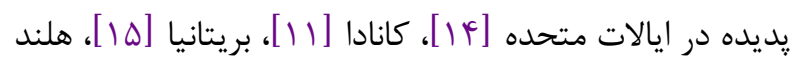

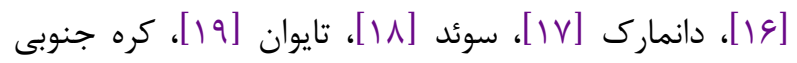

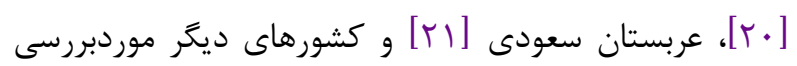

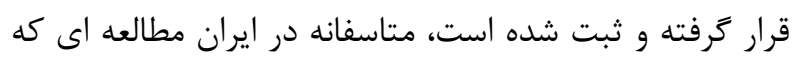

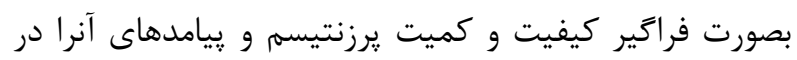
تروههاى وسيع شغلى بررسى كند صورت نكرفته است. در ايران

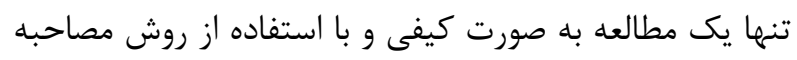

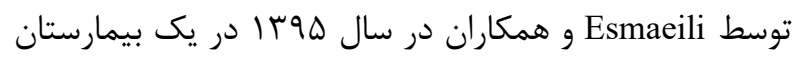

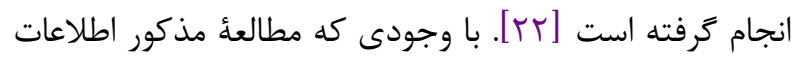

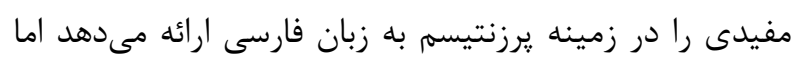

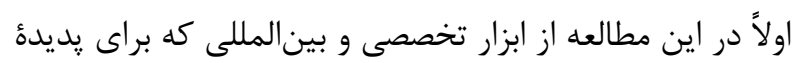

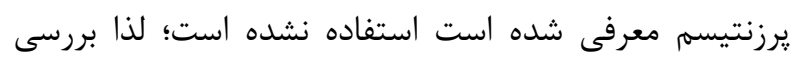

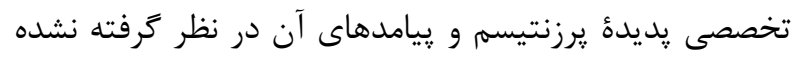

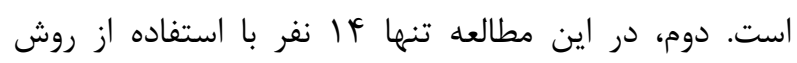

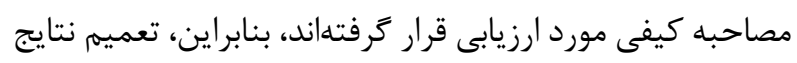

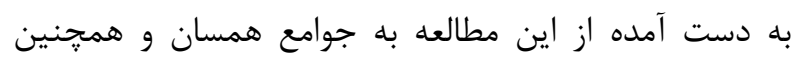

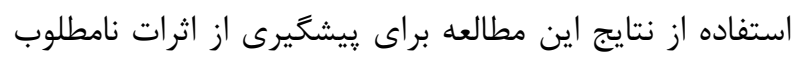

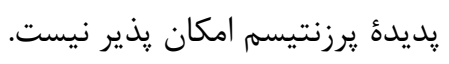

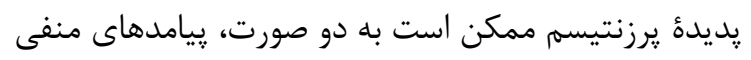

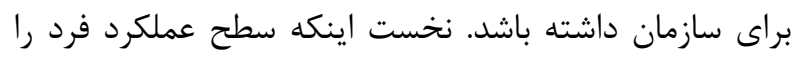

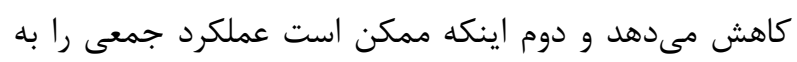

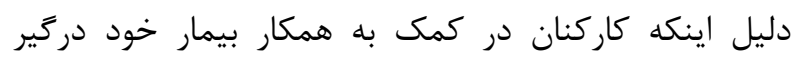

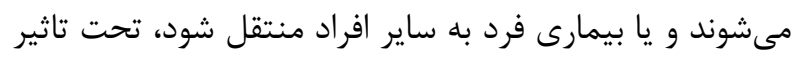

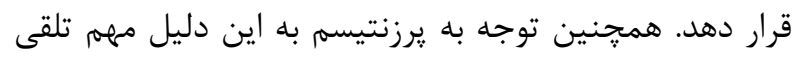

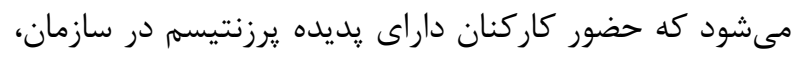

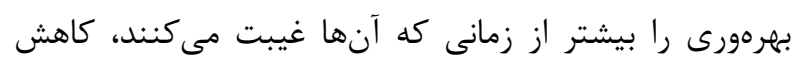

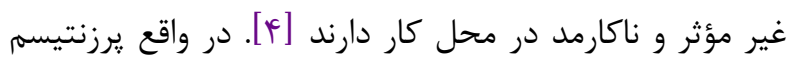

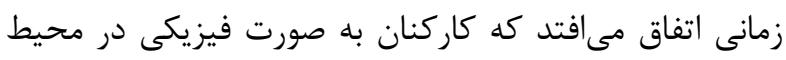

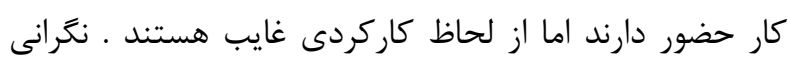

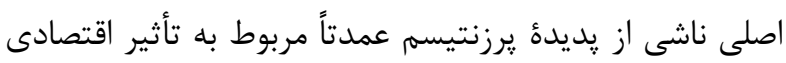

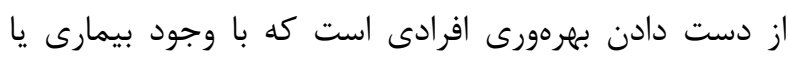

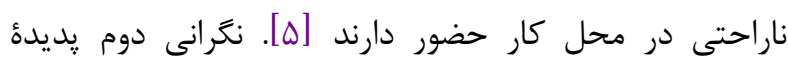

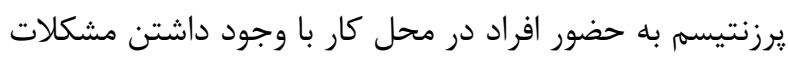

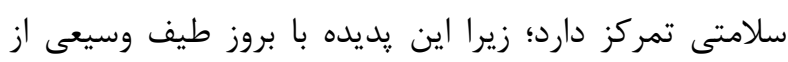

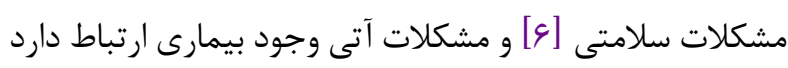

يديدة گرزنتيسم به يك يديده شايع و قابل توجه در

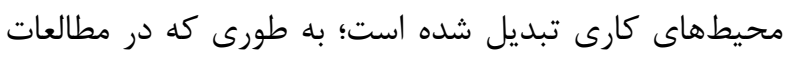

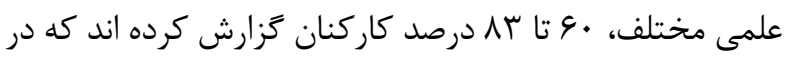

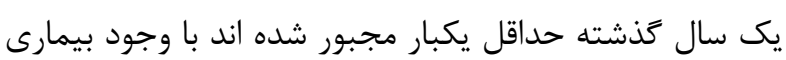

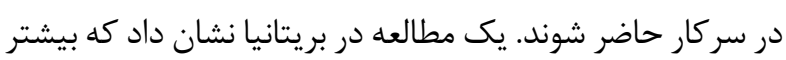

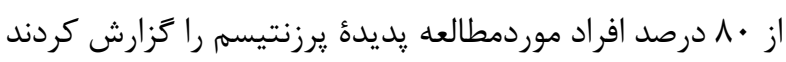

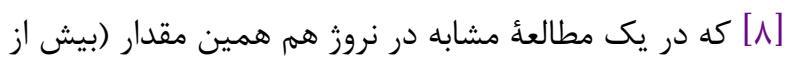

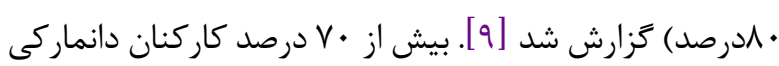

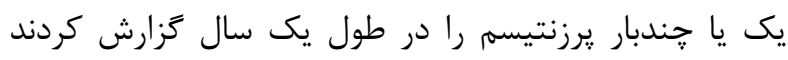

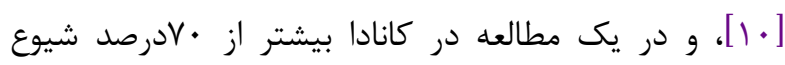

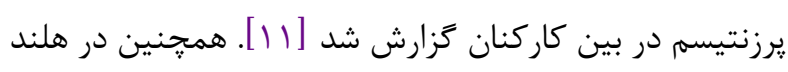

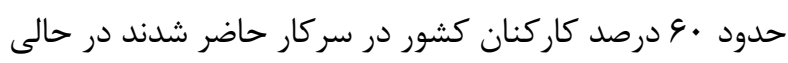

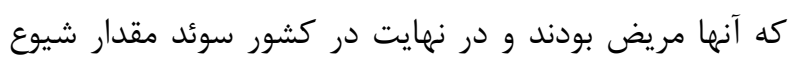

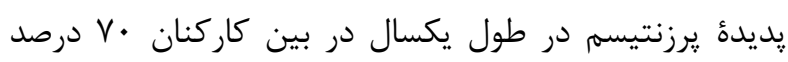

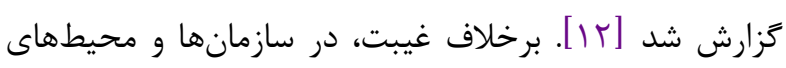

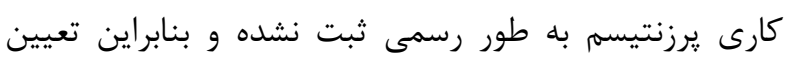

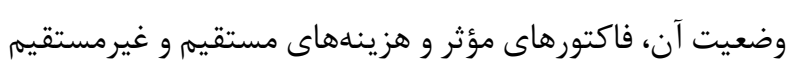

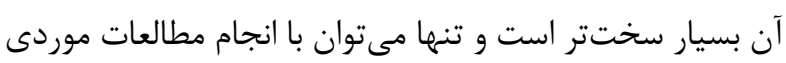
شيوع اين بديده را تزارش كرد.

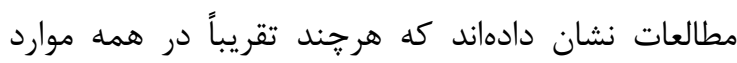

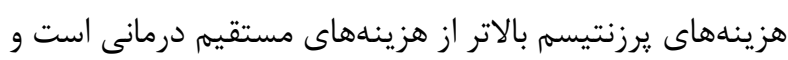

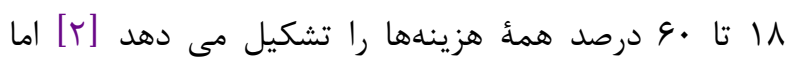

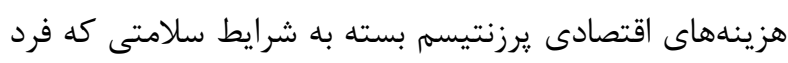

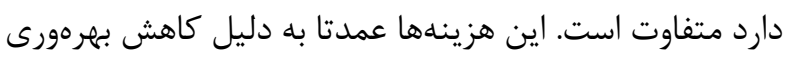

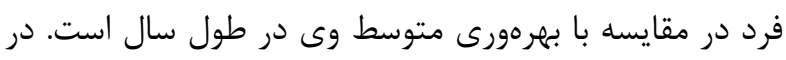

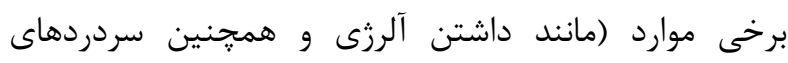
ميخرنى) هزينه يرزنتيسم بسيار بيشتر از هزينهان إنهاى مستقيم 
بومىسازى شده بود. بخش دوم و سوم مطالعه به ترتيب شامل

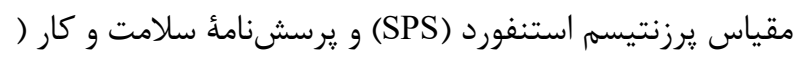
بود. (HWQ)

\section{معرفى مقياس دانشگاه استنفورد (SPS): مقياس}

يرزنتيسم استنفورد (SPS) براى اولين بار توسط

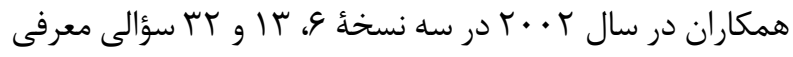
شد. با بررسى سه نسخء SPS، اين محققين در نهايت ششسؤالى (SPS-6) را به عنوان يك مقياس داراى مشخصات روانسنجى عالى معرفى كردند كه امكان استفاده در سنجش إنش

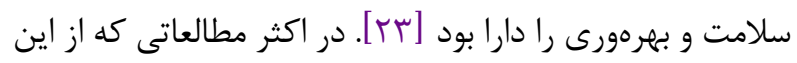
ابزار استفاده كرده اند، مخصوصا در مطالعاتى كه در ايالات متحده انجام شده است، همعى از - 9SPS استفاده كردهاند.

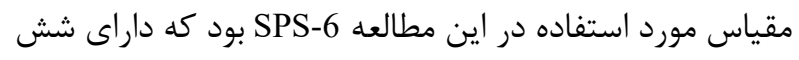

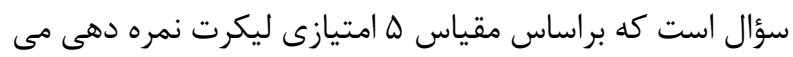

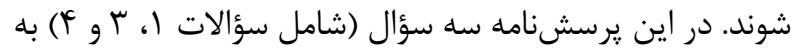

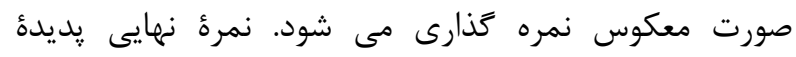

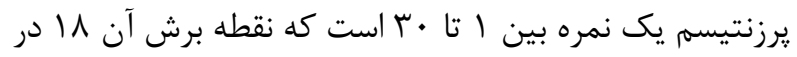

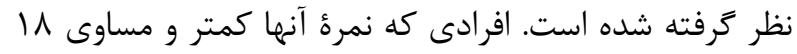

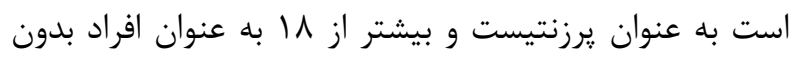

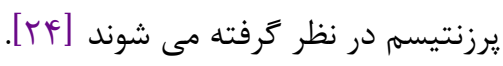

\section{معرفى برسشنامهٔ سلامت و كار (HWQ): برسشنامة}

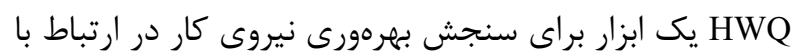

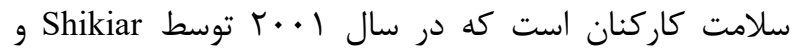

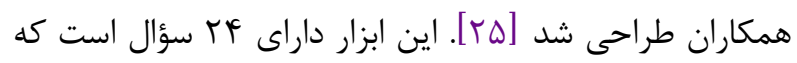

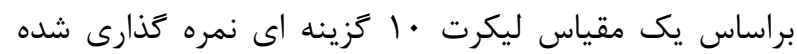

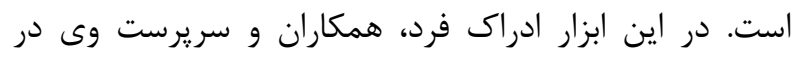

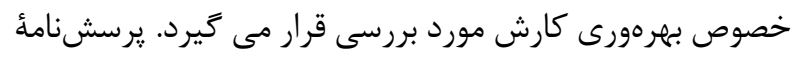

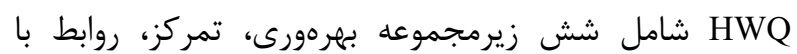
مسئول يا رئيس، بىصبرى و تحريكيذيرى، رضايت در محيط

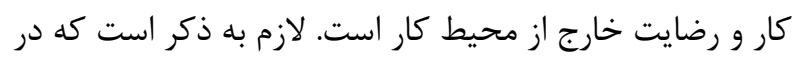

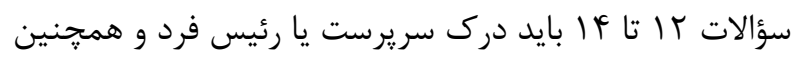
همكاران وى در مورد بهرهورى فرد مورد مطالعه سنجيده شود.

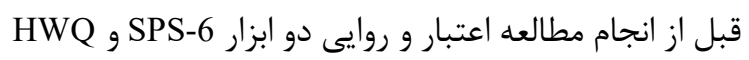

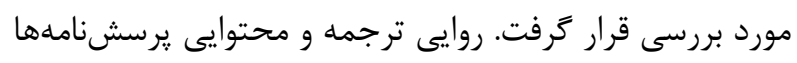

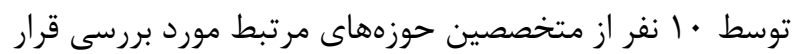

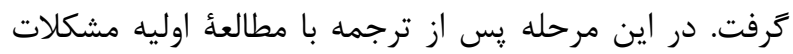

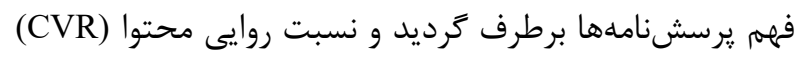

مىدهد؛ بنابراين مديريت مؤثر اين يديده مىتواند به حفظ و ارتقاى سلامت فردى و سلامت سازمان كمك شايانى كند. بنابر اين مطالعه حاضر با هدف بررسى يديدة يرزنتيسمه و ارتباط

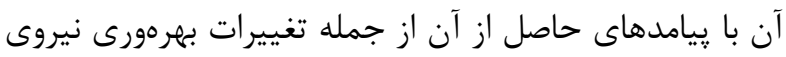
انسانى در بين كاركنان شركت انتقال كاز ايران انجام شد.

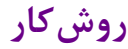

اين مطالعه به صورت مقطعى در بين كاركنان شركت انتقال

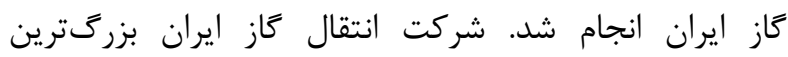

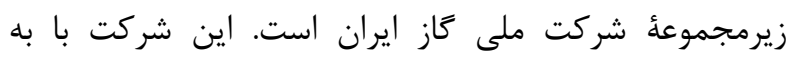

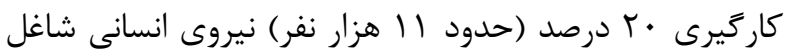

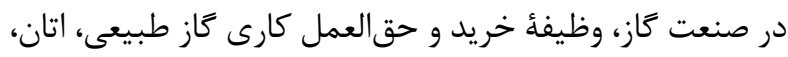

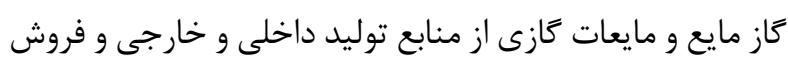

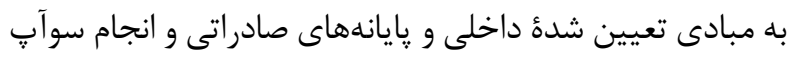

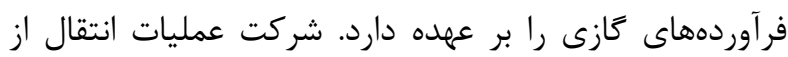

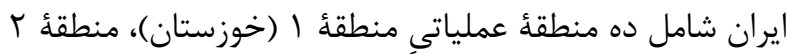

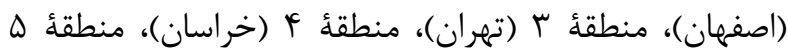

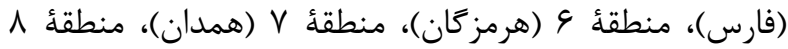

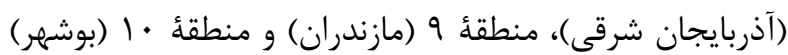
با استفاده از فرمول كوكران و با شرط مشخص بودن جامعل

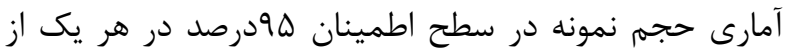

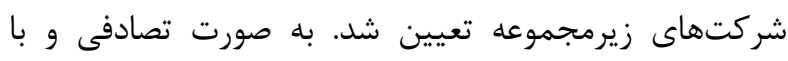

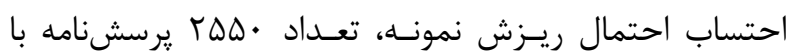

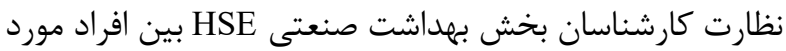

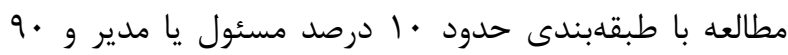

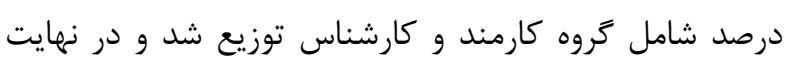

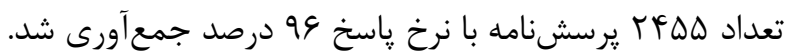

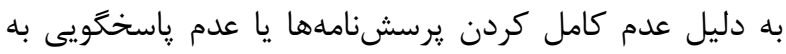

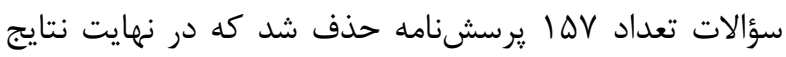

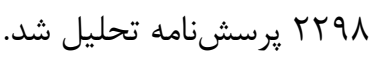
ابزار مطالعه شامل معرفىنامئ مطالعه، رضايتنامه و

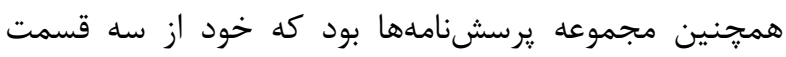
تشكيل شده بود. بخش اول شامل مشخصات فردى مانند سن، جنس، تحصيلات و ... بود كه با هدف جمعآورى مشخصات دموكر افيك و مشخصههاى شغلى كاركنان موردمطالعه تنظيم شده بود. اين سؤالات براساس متون مرتبط جمعآ مآورى و موريكان 


\section{يافته ها}

نتايج مطالعه نشان داد كه ميانگين سنى افراد مورد مطالعه

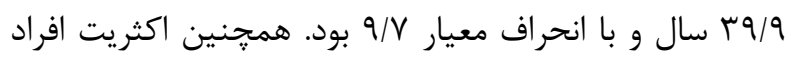

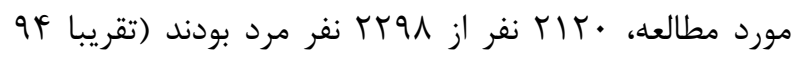

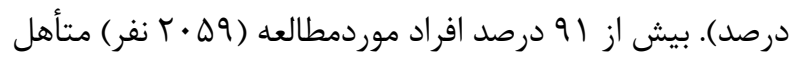

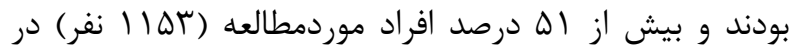

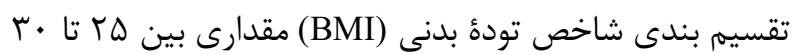

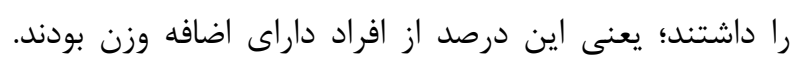

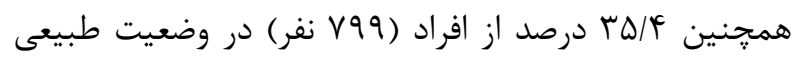

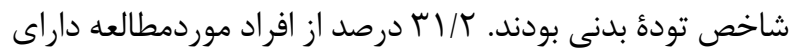

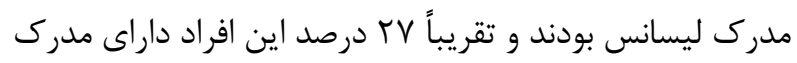
فوق ليسانس بودند. هل درصد از افراد موردمطالعه در عمليات

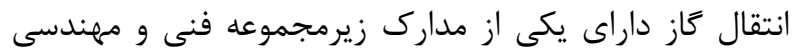
بودند و بيش از F T درصد داراى مدرى علوم انسانى بودند.

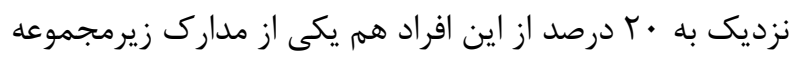

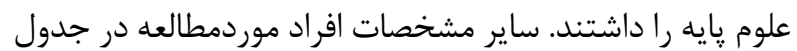

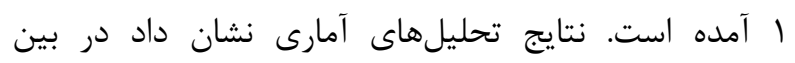

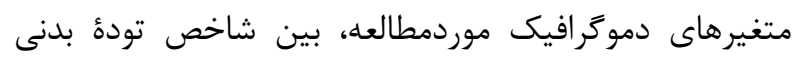

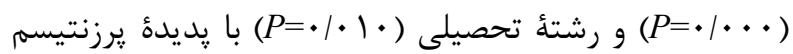

$$
\text { از نظر آمارى ارتباط معنادارى وجود داشت. }
$$

و شاخص روايى محتوا (CVI) براى ابزار SPS-6 به ترتيب 9 •

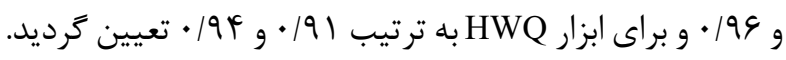
همجنين :ايايى يرسشنامهها با استفاده از ضريب آلفاى كرونباخ

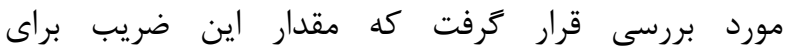

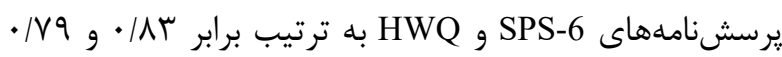

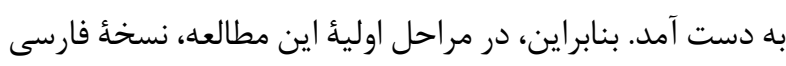

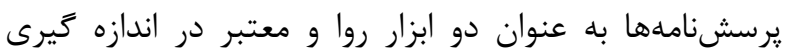
يرزنتيسم و بهرهورى نيروى انسانى يذيرفته شدند.

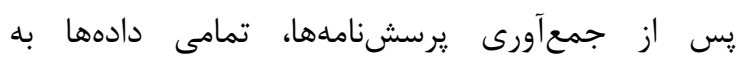

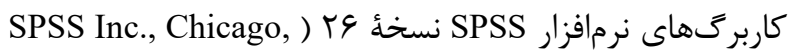
(Ill., USA فراوانى و انحراف استاندارد از آمار توصيفى استفاده شد. براى بـى دئ

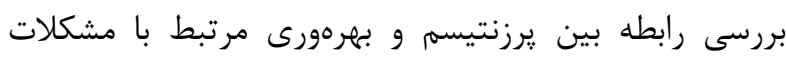

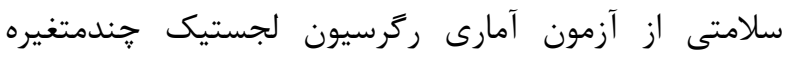

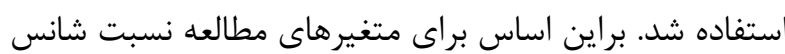

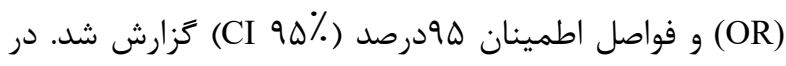

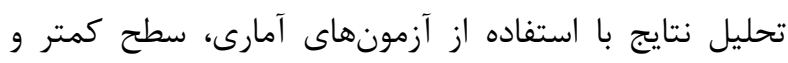

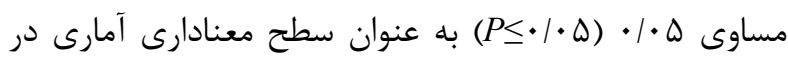
نظر زرفته شد.

جدول ا: مشخصات فردى (متغيرهاى دموكرافيك) و مشخصات شغلى كاركنان شركت انتقال كاز ايران

\begin{tabular}{|c|c|c|c|c|}
\hline $\boldsymbol{P}^{*}$ & درصد فراوانى & ميانَين (土انحراف معيار) / فراوانى & \multicolumn{2}{|c|}{ متغير } \\
\hline .1119 & - & ४q/q) $\pm 9 / 9 V$ & \multicolumn{2}{|c|}{ سن (سال) } \\
\hline \multirow{2}{*}{$\cdot 11 \cdot 4$} & $9 \pi / V$ & TIT. & 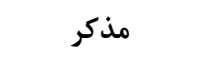 & \multirow{2}{*}{ 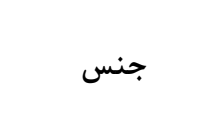 } \\
\hline & g/r & IFr & مونث & \\
\hline \multirow{2}{*}{.$/ 91 F$} & $1 / 9$ & $r \cdot 1$ & 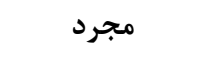 & \multirow{2}{*}{ 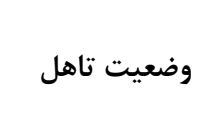 } \\
\hline & $91 / 1$ & $r \cdot \Delta q$ & 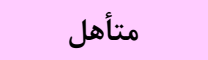 & \\
\hline$\cdot 1 \cdot \operatorname{tr}$ & - & $1 / V T \pm \cdot / Y F$ & قد (متر) & \multirow{2}{*}{ 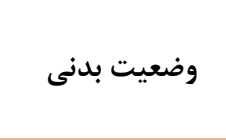 } \\
\hline$\cdot 1 \cdots$ & - & $\Lambda \cdot / V \cdot \pm 1 r / \Lambda 9$ & وزن (كيلو ترم) & \\
\hline \multirow{5}{*}{$\cdot / \cdots$} & $\cdot / \Lambda$ & 11 & 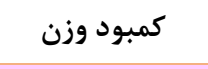 & \multirow{5}{*}{ شاخص توده بدنى } \\
\hline & $r \Delta / \boldsymbol{q}$ & V१९ & طبيعى & \\
\hline & $\Delta 1 / 1$ & llar & 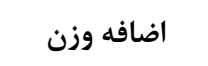 & \\
\hline & $11 \%$ & TFA & 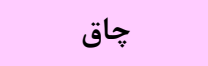 & \\
\hline & $1 / V$ & $r q$ & خيلى جاق & \\
\hline
\end{tabular}




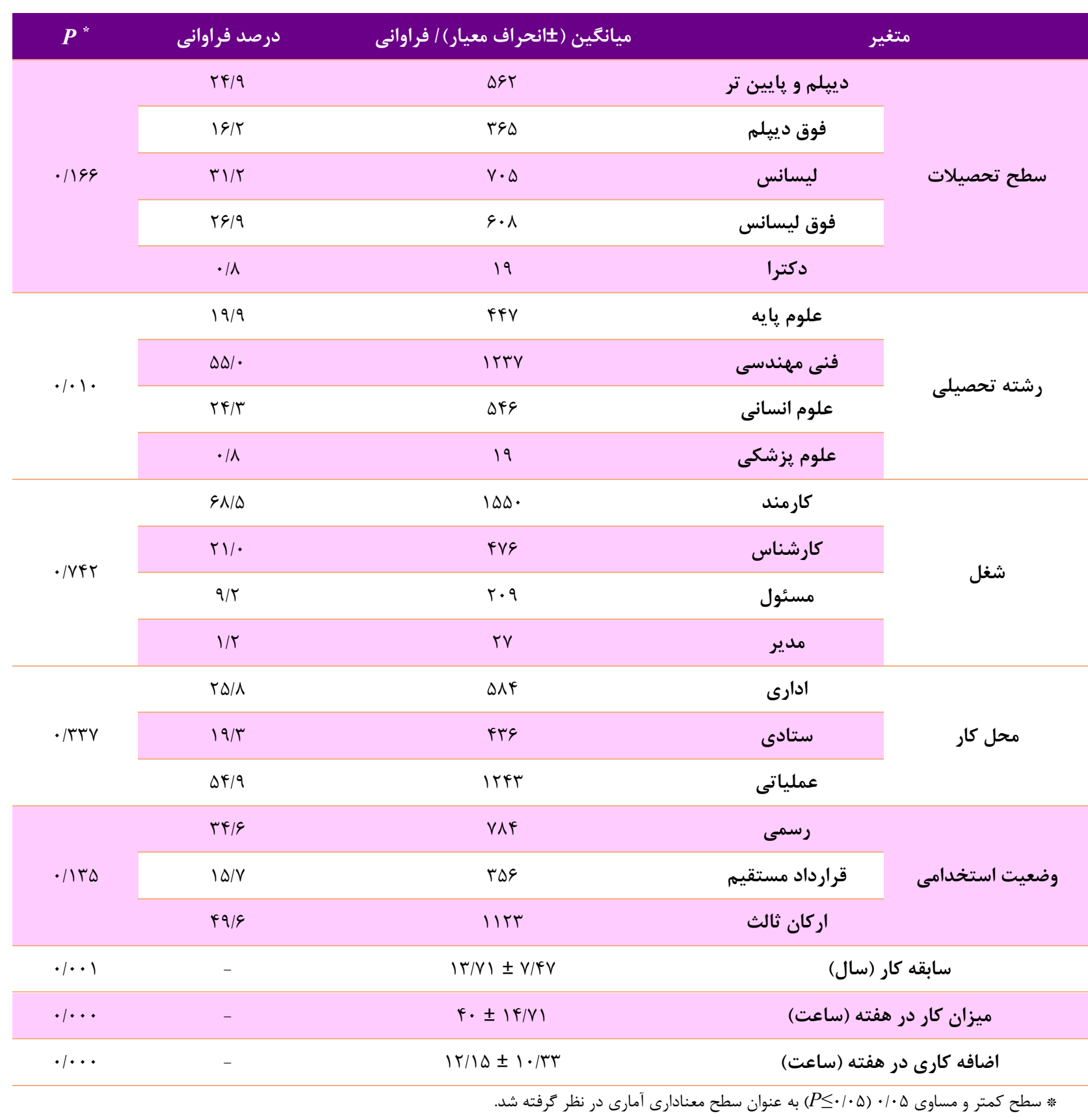

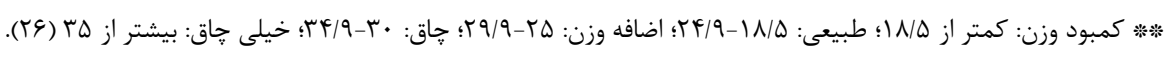

و قرارداد مستقيم با ه/V إ درصد قرار داشتند. ميانگين سابقه

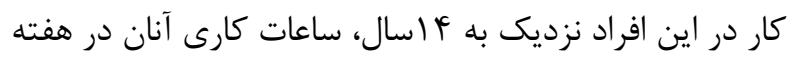

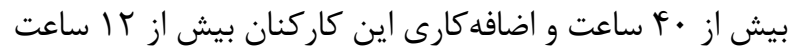

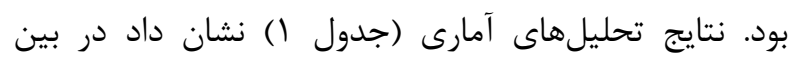

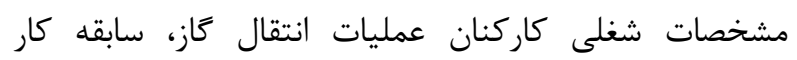

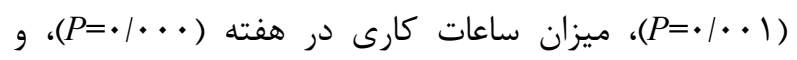

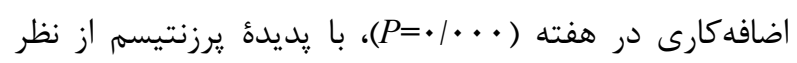
آمارى رابطه معنادارى وجود داشت.
بيش از 91 درصد كاركنان مورد مطالعه در شركت انتقال

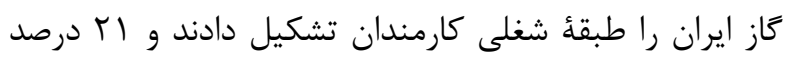

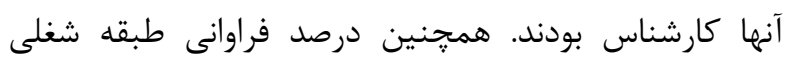

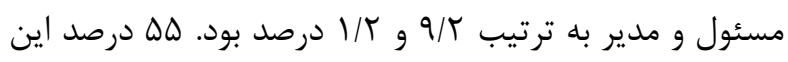

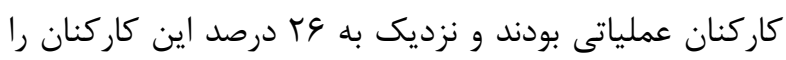

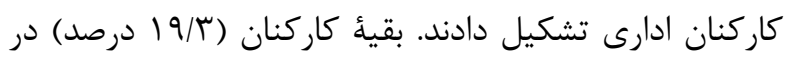
قسمتهاى ستادى كار مى كردند. بيشتر كاركنان موردمطالعه

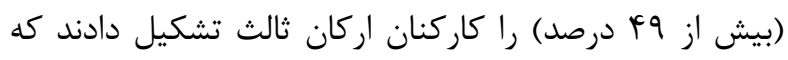

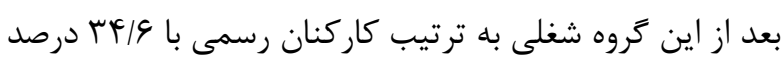




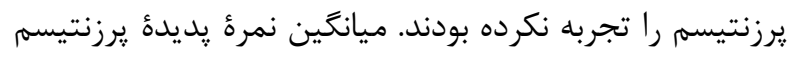

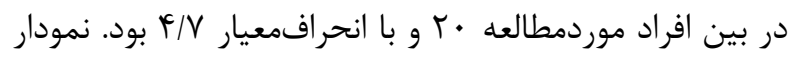

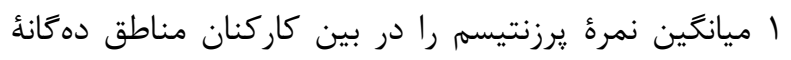

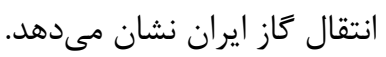

مطالعه وضعيت يرزنتيسم در بين كل كاركنان عمليات

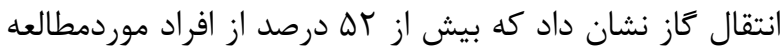

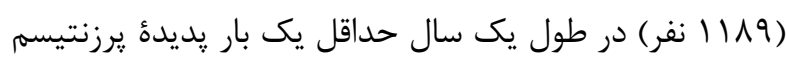

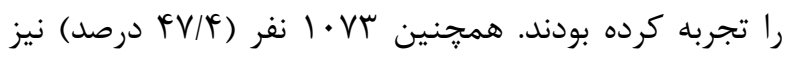

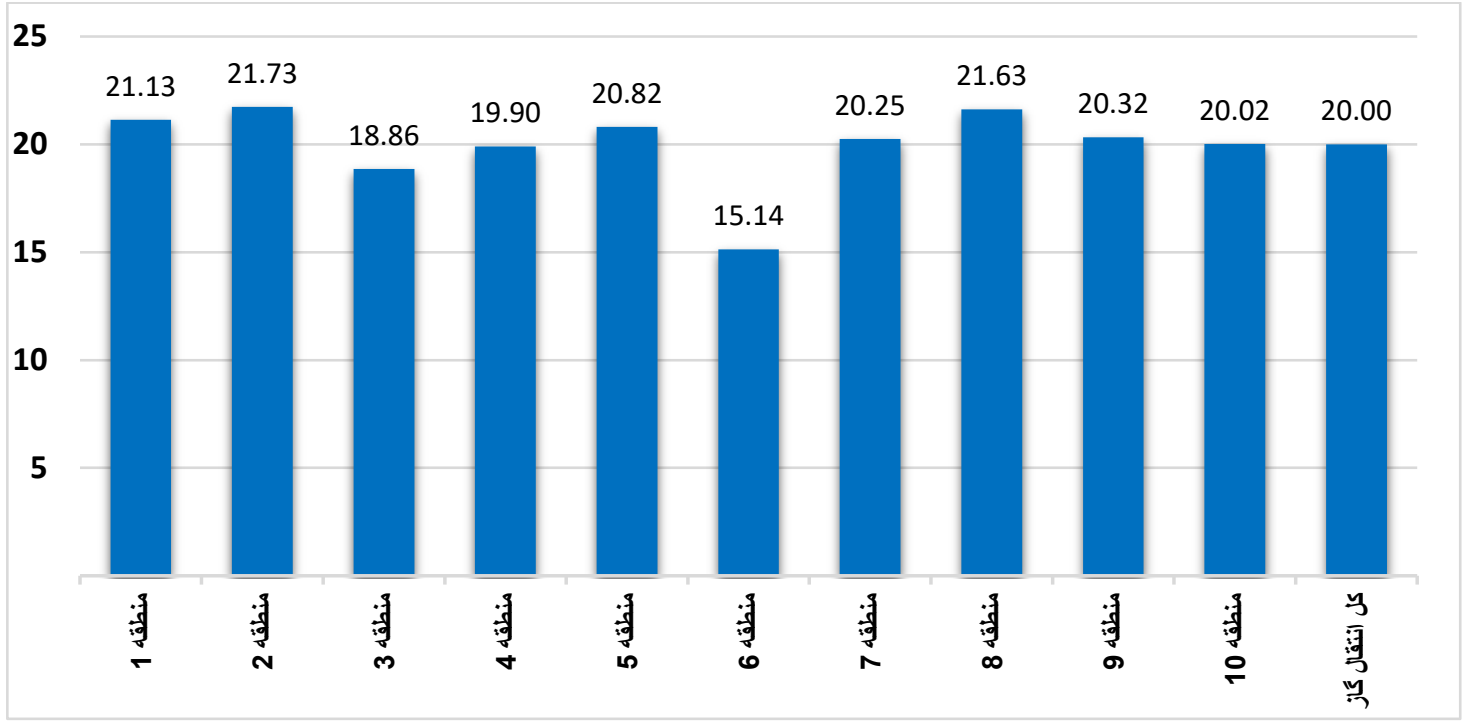

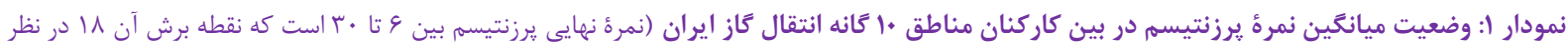

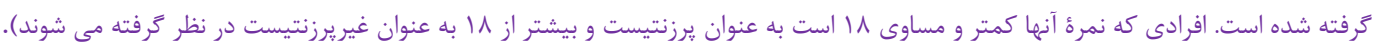

غيريرزنتيست بود. نتايج حاصل از تحليل آزمون ركرسيون

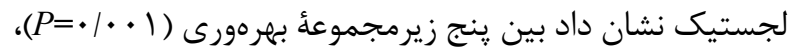

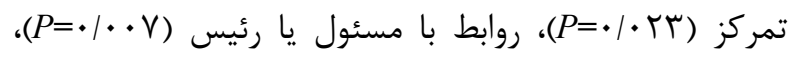

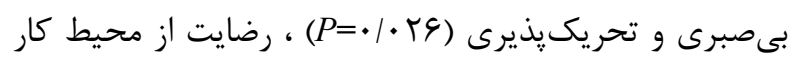

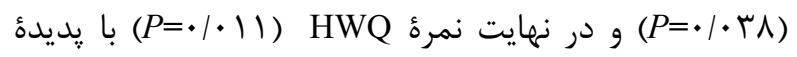

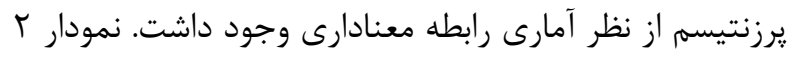

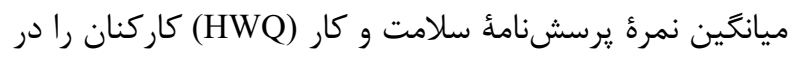

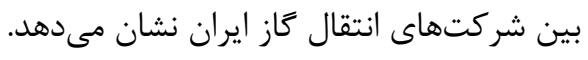

همانطور كه نتايج جدول r نشان مى دهد در دو زيرمجموعة

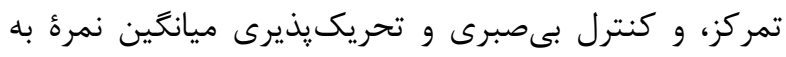

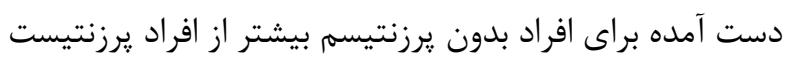

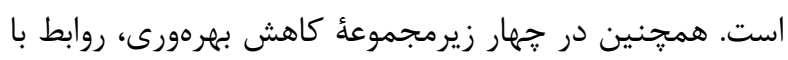

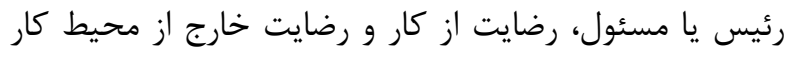

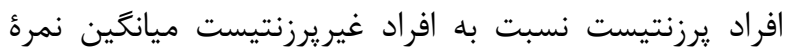

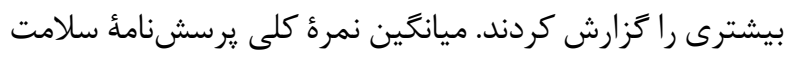

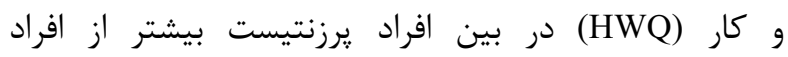

جدول r: ميانَين (ذانحراف معيار) وضعيت مقياس سلامت و كار و وزير مجموعه آن در بين كاركنان شركت انتقال كاز ايران

\begin{tabular}{|c|c|c|c|c|c|c|c|}
\hline \multirow{2}{*}{$\boldsymbol{P}^{*}$} & \multicolumn{2}{|c|}{ كل افراد موردمطالعه } & \multicolumn{2}{|c|}{ بدون برزنتيسم } & \multicolumn{2}{|c|}{ داراى برزنتيسم } & \multirow{2}{*}{ زير مجموعه ها } \\
\hline & SD & ميانكين & SD & ميانغين & ${ }^{* *}$ SD & 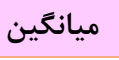 & \\
\hline$\cdot 1 \cdot \cdot 1$ & $r F / l$ & $\vee 9 / .9$ & rT/GG & $V V / I$ & TY/Tr & $\Lambda \cdot \mid \Lambda F$ & بهرهورى \\
\hline ש & $\Delta / \Gamma I$ & $\mid \Delta / \wedge \Lambda$ & $F / 90$ & $19 / T r$ & $\Delta / 9 \mid$ & $1 \Delta / \Delta V$ & تمركز \\
\hline$\cdot / \cdot V$ & $F / 1$ & $19 / 49$ & $f /$. & $19 / \pi 4$ & $f / 1 Q$ & $19 / V$ & روابط با مسئول / رييس \\
\hline . & $V / V \Delta$ & IT/AF & V/AT & $1 \pi / 9 \Lambda$ & V/৭६ & $|r /|$ & بى صبرى و تحريك يذيرى \\
\hline$\cdot \mid \cdot r \wedge$ & $V / T V$ & $r r / 9 q$ & $9 / 9 V$ & Tr/Tr & V/GF & 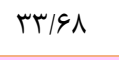 & رضايت از كار \\
\hline$\cdot / r \Delta \Lambda$ & S/AD & $r \Delta / \Delta \cdot$ & $N / f \mid$ & $r \Delta / r \Delta$ & $\Delta / \cdot r$ & $r \Delta / V I$ & رضايت خارج از محيط كار \\
\hline$\cdot 1 \cdot 11$ & rV/A & $|V q / V|$ & rQ/VQ & $11 \cdot 109$ & $r 1 / \cdot r$ & $\mid \Lambda F / F$ & HWQ \\
\hline
\end{tabular}

* سطح كمتر و مساوى ه • • (PS0.05) به عنوان سطح معنادارى آمارى در نظر كرفته شد؛ 


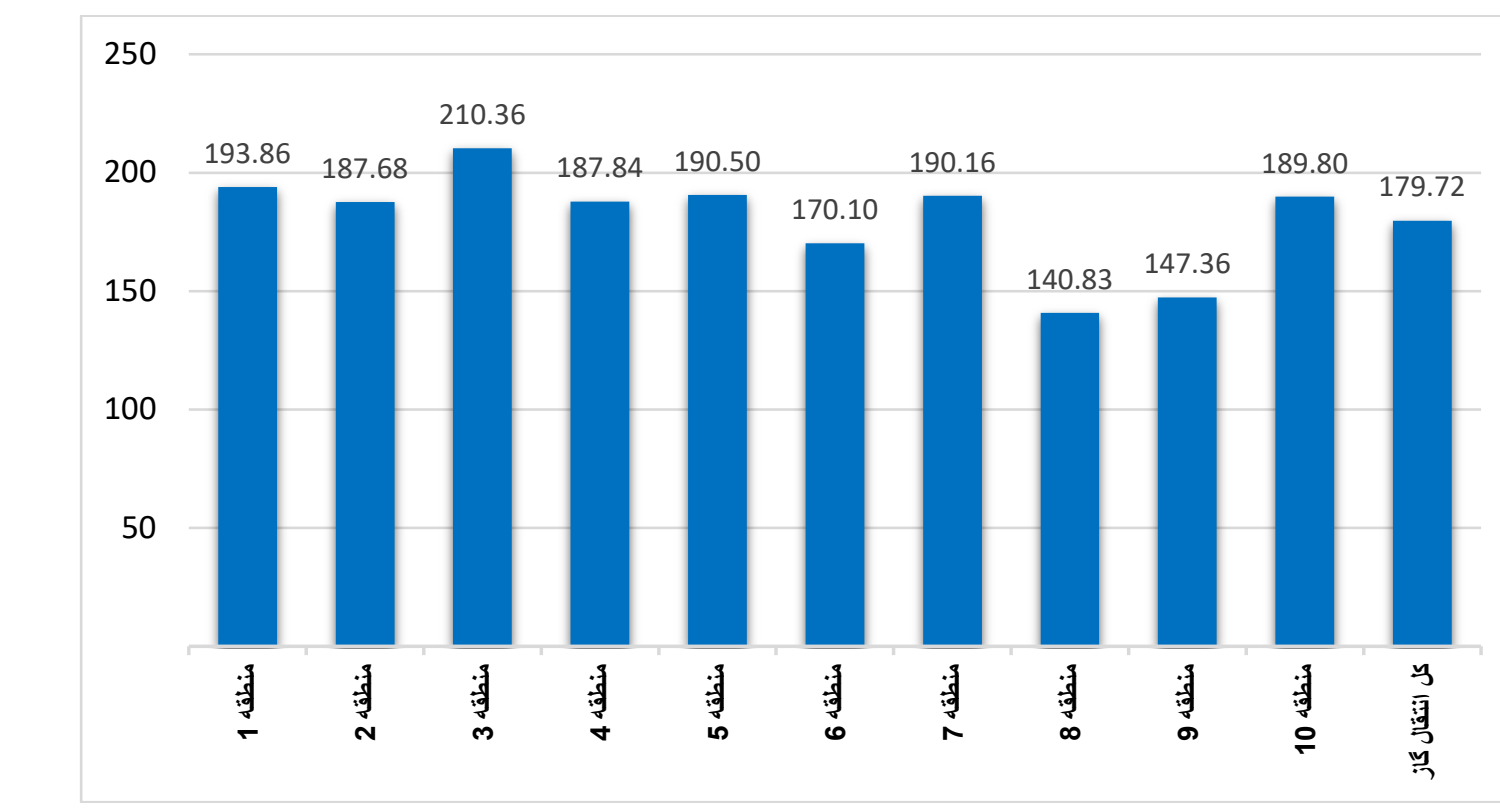

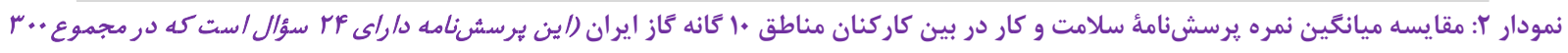
نمره د/رد).

همكاران در يك مطالعه ارتباط بين شاخص توده بدنى و بهرهورى

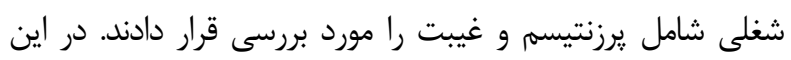

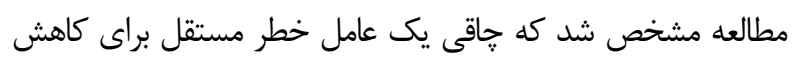

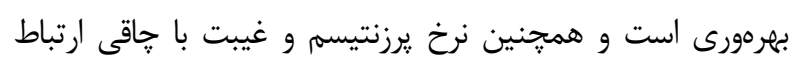

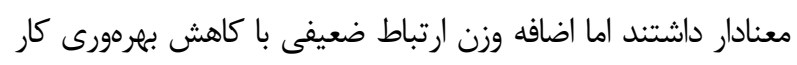

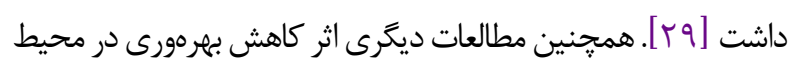

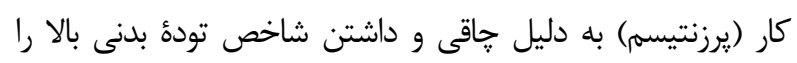

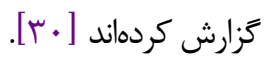
اين مطالعه نشان داد بين نرخ شيوع يرزنتيسم و سه مشخصأ سأن

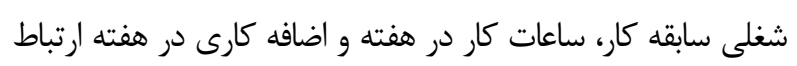

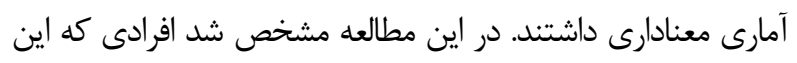

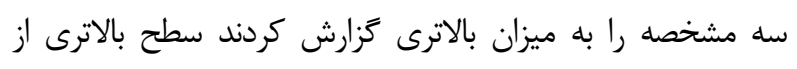

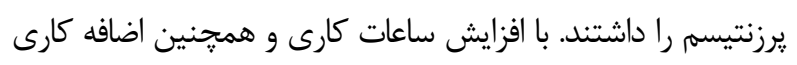
در طول هفته شخص دستيابى به سطح درآمدى بيشترى را دنبال

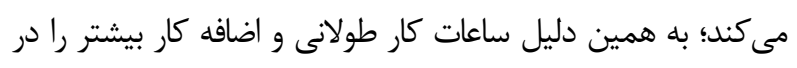

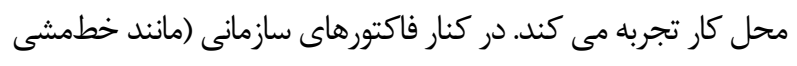

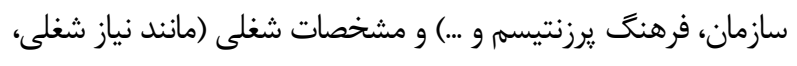

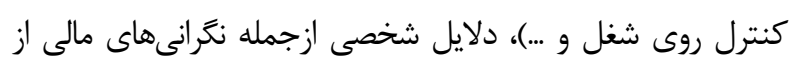

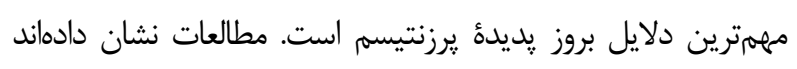
استرسهاى مالى ازجمله كاهش حقوق و سطح درآمد فرد يكى از

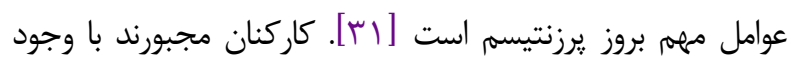
بيمارى در محل كار حضور داشته باشند زيرا در درجهُ اول آنان به دليل
در اين مطالعه بديدة يرزنتيسم و امكان اثر آن بر كاهش بهرهورى

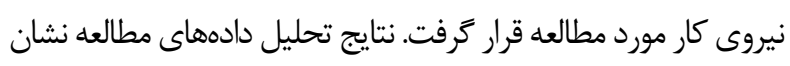

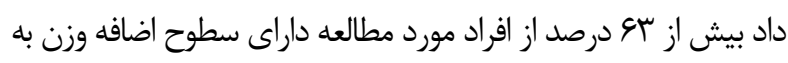

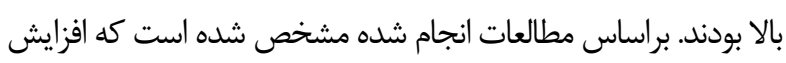

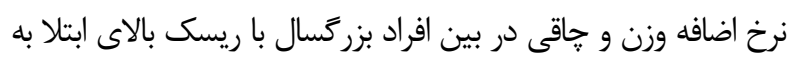

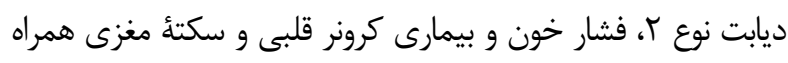

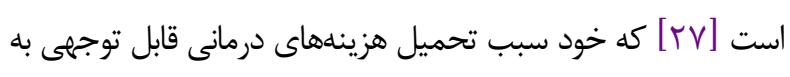

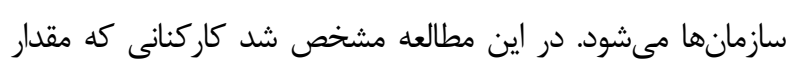

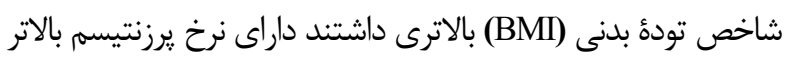

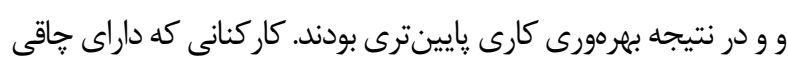

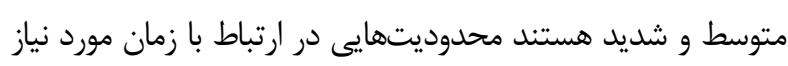

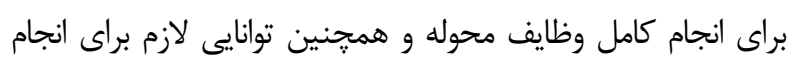
نيازهاى فيزيكى شغل دارند، در حالى كه كاركنان ديكر (افراد طبيعى إنى

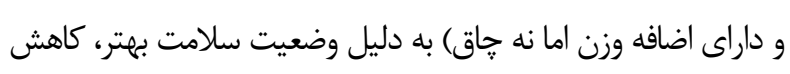

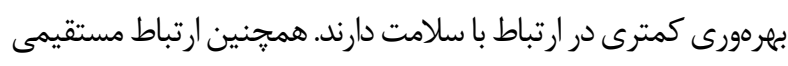

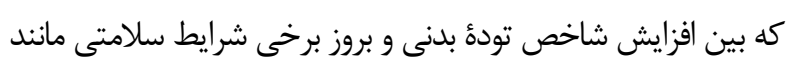

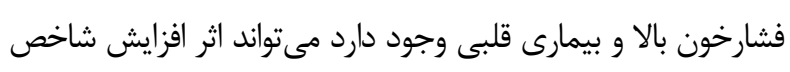

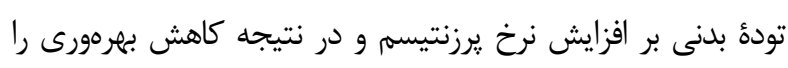

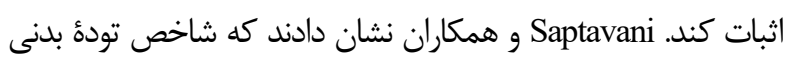

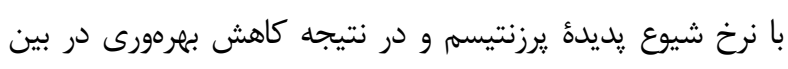

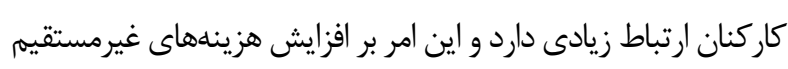

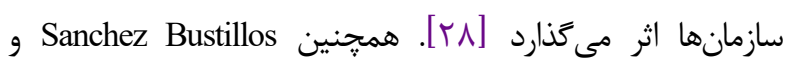


دفعات آن در بين كاركنان دارد. همسو با اين نتايج، جندين مطالعه

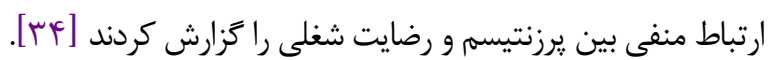
Goetzel و همكاران تخمين زدند كه هزينههاى يرزنتيسم بلهطور

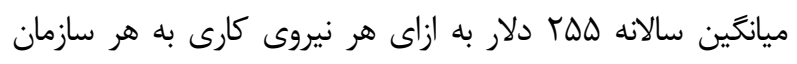

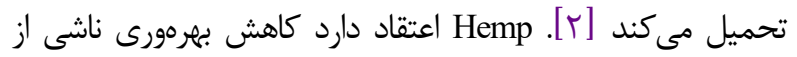

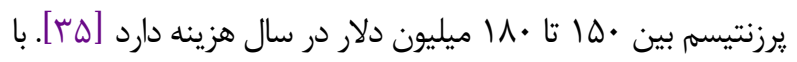

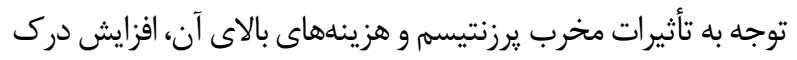

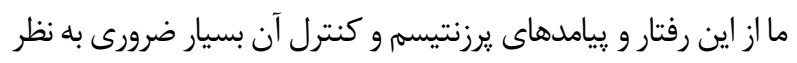

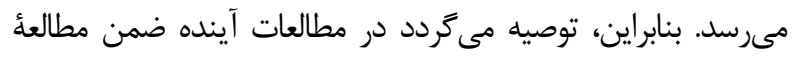

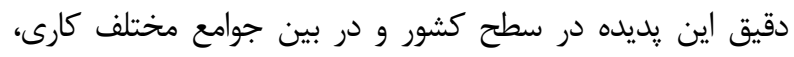

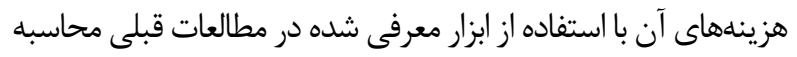
و كنترل كردد. براساس نتايج اين مطالعه، اجراى برنامههاى كنترل

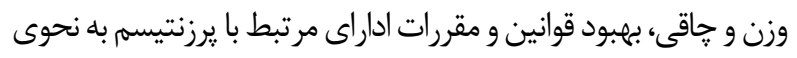

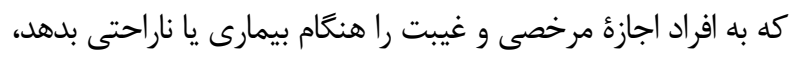

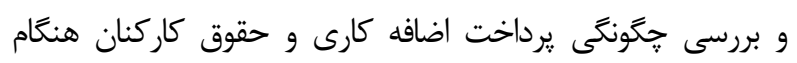
مرخصى استعلاجى يا دوركارى براى كاهش يرزنتيسم و هزينه آنهاف آن توصيه مىشود. يكى از متغيرهايى كه يديدة يرزنتيسم روى آن آن اثر كذاشت

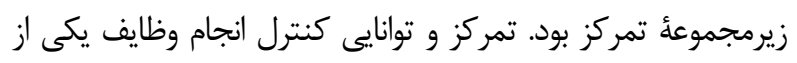
اركان اصلى انجام صحيح كار است. عدم وجود تمركز كافى مى توتواند

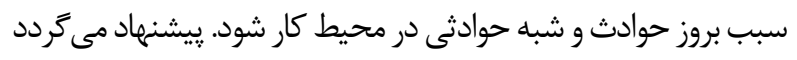

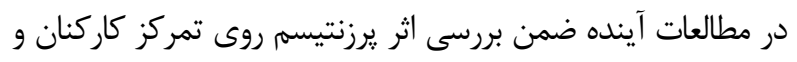

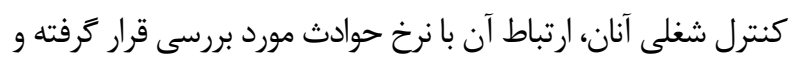
راهكارهاى كنترلى ارائه و اجرا شود.

\section{نتيجه گَيرى}

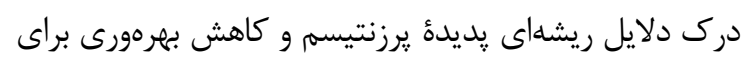

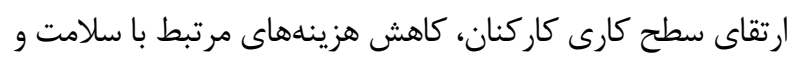
ثبات شركتها امرى ضرورى است. اين مطالعه نشان داد كه بر بروز

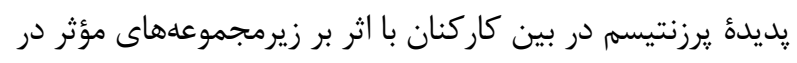

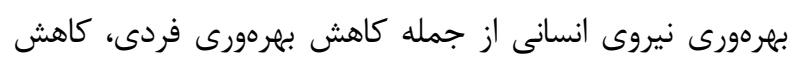

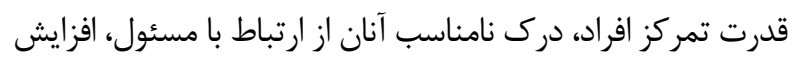

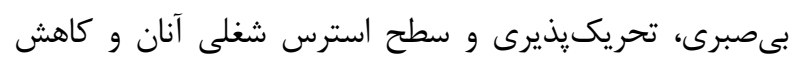

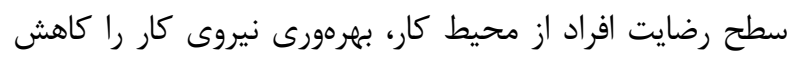

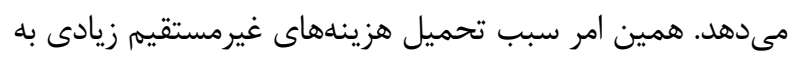

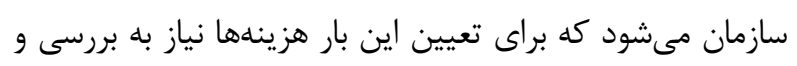

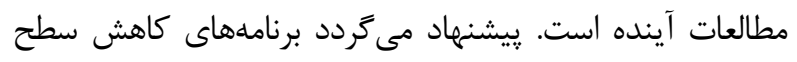

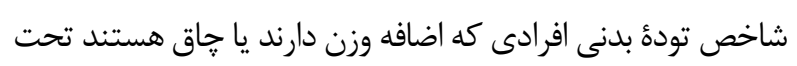

تأمين هزينههاى زندگى و حمايت از خانواده نمىتوانند كاهش حقوق

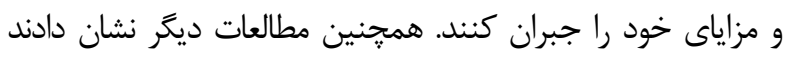
سطوح بالاى نيازهاى زمانى در محيط كار ازجمله اضافه كار، ساعات

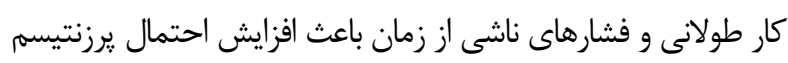

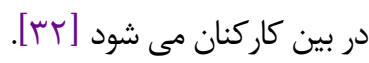

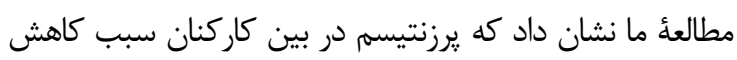

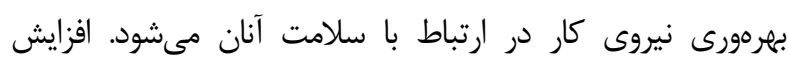

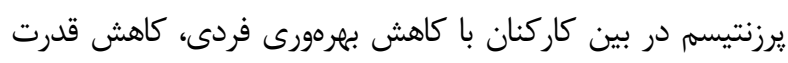

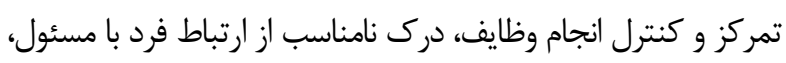

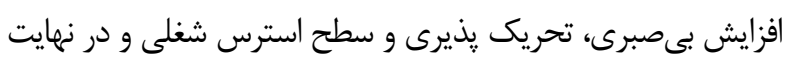

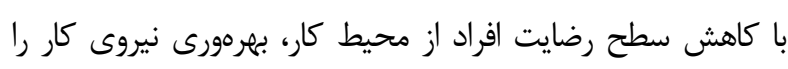

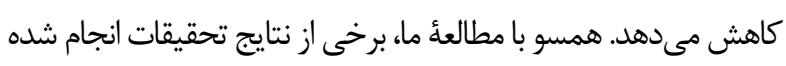

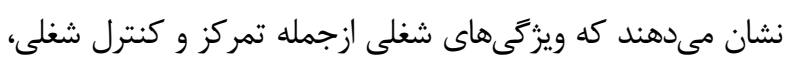

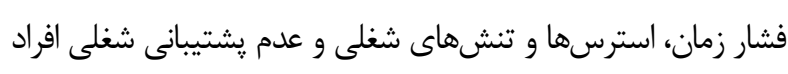

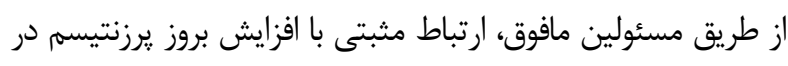

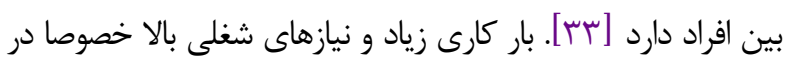

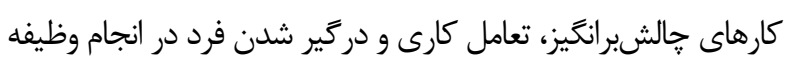

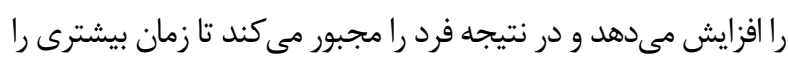

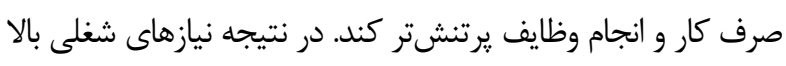

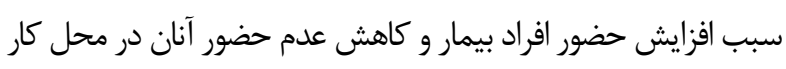

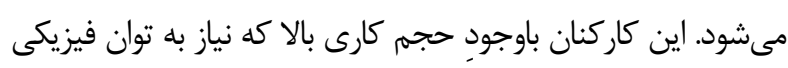

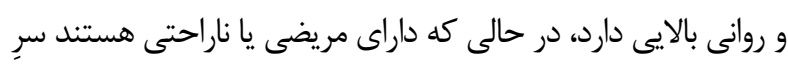

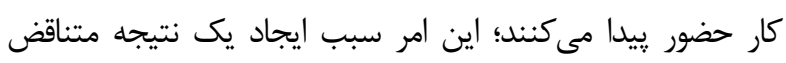

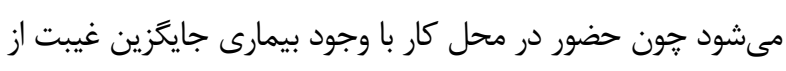

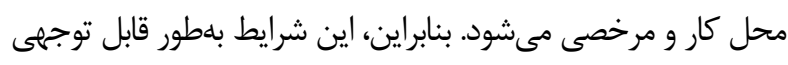

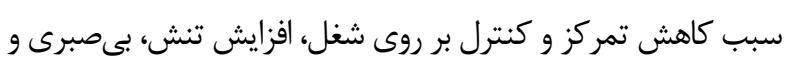

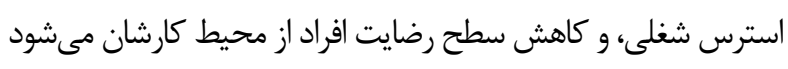

در اين مطالعه مشخص شد بين بديدة برزنتيسم, وضايت شغلى،

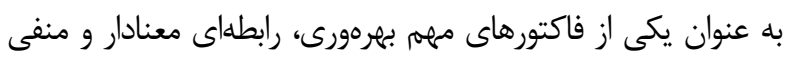

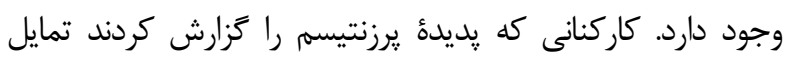

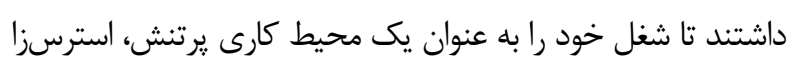

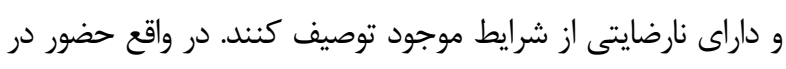

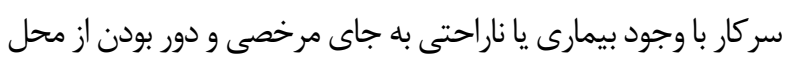

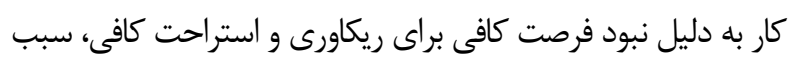

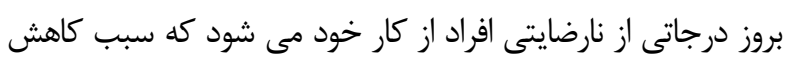

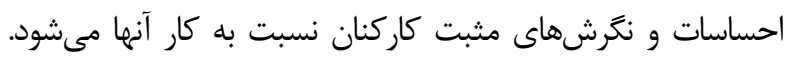

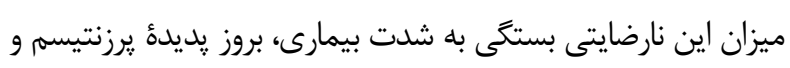




$$
\begin{aligned}
& \text { شركت انتقال كاز ايران كه در كردآورى دادهها مشاركت فعال } \\
& \text { داشتند تشكر و قدردانى مىنمايند. }
\end{aligned}
$$

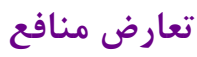

$$
\begin{aligned}
& \text { بين نويسندكان هيجَّونه تعارضى در منافع وجود ندارد. } \\
& \text { منابع مالى } \\
& \text { منابع مالى اين مطالعه توسط شركت ملى كاز ايران تامين شده } \\
& \text { نظر متخصصان تغذيه و رزيمدرمانى اجرا شود. همجنين اصلاح } \\
& \text { قوانين و مقررات ادارى توصيه مى گردد به گونهاى كه با استفاده از }
\end{aligned}
$$

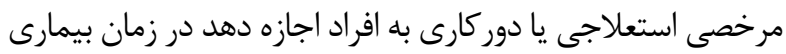

$$
\begin{aligned}
& \text { يا ناراحتى در محل كار حضور نداشته باشند. } \\
& \text { تقدير و تشكر } \\
& \text { اين مطالعه نتيجهُ قسمتى از يك برنامهٔ عملياتى اجراشده } \\
& \text { در سطح شركت ملى گاز ايران است. بدين وسيله نويسند } \\
& \text { زحمات مسئولين و كارشناسان محترم بهداشت صنعتى و HSE }
\end{aligned}
$$

\section{References}

1. Bierla I, Huver B, Richard S. New evidence on absenteeism and presenteeism. Int J Hum Resour Manag. 2013;24(7):1536-50. [DOI:10.1080/09585192.2012.722120]

2. Goetzel RZ, Long SR, Ozminkowski RJ, Hawkins K, Wang S, Lynch W. Health, absence, disability, and presenteeism cost estimates of certain physical and mental health conditions affecting US employers. J occup Environ Med. 2004;46(4):398-412.

[DOI:10.1097/01.jom.0000121151.40413.bd] [PMID]

3. Gosselin E, Lemyre L, Corneil W. Presenteeism and absenteeism: Differentiated understanding of related phenomena. J Occup Health Psychol. 2013;18(1):75-86. [DOI:10.1037/a0030932] [PMID]

4. Widera E, Chang A, Chen HL. Presenteeism: a public health hazard. J Gen Intern Med. 2010;25(11):1244-7. [DOI:10.1007/s11606-010-1422-x] [PMID] [PMCID]

5. Johns G. Presenteeism in the workplace: A review and research agenda. J Organ Behav. 2010;31(4):519-42. [DOI: 10.1002/job.630]

6. Skagen K, Collins AM. The consequences of sickness presenteeism on health and wellbeing over time: A systematic review. Soc Sci Med. 2016;161:169-77 [DOI:10.1016/j.socscimed.2016.06.005] [PMID]

7. Bergström G, Bodin L, Hagberg J, Aronsson G, Josephson M. Sickness presenteeism today, sickness absenteeism tomorrow? A prospective study on sickness presenteeism and future sickness absenteeism. J Occup Environ Med. 2009;51(6):629-38.

[DOI:10.1097/JOM.0b013e3181a8281b] [PMID]

8. McKevitt C, Morgan M, Dundas R, Holland W. Sickness absence and 'working through'illness: a comparison of two professional groups. J Public Health. 1997;19 (3): 295-300. [PMID] [DOI:10.1093/oxfordjournals.pubmed.a024633]

9. Rosvold EO, Bjertness E. Physicians who do not take sick leave: hazardous heroes? Scand J Public Health. 2001;29(1):71-5. [DOI: $10.1177 / 14034948010290010101]$

10. Hansen CD, Andersen JH. Going ill to work-What personal circumstances, attitudes and work-related factors are associated with sickness presenteeism? Soc Sci Med. 2008;67(6):956-64

[DOI:10.1016/j.socscimed.2008.05.022] [PMID]

11. Caverley N, Cunningham JB, MacGregor JN. Sickness presenteeism, sickness absenteeism, and health following restructuring in a public service organization. J Manag Stud. 2007;44(2):304-19. [DOI:10.1111/j.1467-
[D. 6486.2007.00690.x]

12. Aronsson G, Gustafsson K. Sickness presenteeism: prevalence, attendance-pressure factors, and an outline of a model for research. J Occup Environ Med. 2005;47(9):958-66. [DOI:10.1097/01.jom.0000177219.75677.17] [PMID]

13. Lohaus D, Habermann W. Presenteeism: A review and research directions. Hum Resour Manag Rev. 2019;29(1):43-58. [DOI:10.1016/j.hrmr.2018.02.010]

14. Burton WN, Conti DJ, Chen C-Y, Schultz AB, Edington DW. The role of health risk factors and disease on worker productivity. J Occup Environ Med. 1999;41(10):869-77 [DOI:10.1097/00043764-199910000-00007] [PMID]

15. Lu L, Cary LC, Yen Lin H. A cross-cultural examination of presenteeism and supervisory support. Career Dev Int. 2013;18(5):440-56. [DOI:10.1108/CDI-03-2013-0031]

16. Ospina MB, Dennett L, Waye A, Jacobs P, Thompson AH. A systematic review of measurement properties of instruments assessing presenteeism. Am J Manag Care. 2015;21(2):e171-85.

17. Hansen CD, Andersen JH. Going ill to work - What personal circumstances, attitudes and work-related factors are associated with sickness presenteeism? Soc Sci Med. 2008;67(6):956-64.

[DOI:10.1016/j.socscimed.2008.05.022] [PMID]

18. Johansen V, Aronsson G, Marklund S. Positive and negative reasons for sickness presenteeism in Norway and Sweden: a cross-sectional survey. BMJ Open. 2014;4(2):e004123. [DOI:10.1136/bmjopen-2013004123] [PMID] [PMCID]

19. Lu L, Lin HY, Cooper CL. Unhealthy and present: Motives and consequences of the act of presenteeism among Taiwanese employees. J Occup Health Psychol. 2013;18(4):406-16. [DOI:10.1037/a0034331] [PMID] 
20. Kim J, Suh EE, Ju S, Choo H, Bae H, Choi H. Sickness Experiences of Korean Registered Nurses at Work: A Qualitative Study on Presenteeism. Asian Nurs Res. 2016;10(1):32-8. [DOI:10.1016/j.anr.2015.10.009] [PMID]

21. Al Nuhait M, Al Harbi K, Al Jarboa A, Bustami R, Alharbi S, Masud N. et al. Sickness presenteeism among health care providers in an academic tertiary care center in Riyadh. J Infect Public Health. 2017;10(6):711-5. [DOI:10.1016/j.jiph.2016.09.019] [PMID]

22. Esmaeili NM, Ghaedi S, Amiri MY, Gholipour A. Presenteeism Phenomenon: Investigating the Outcomes and the Solutions of Managing it. J Public Adm Perspective. 2016;7(25):67-93.

23. Koopman C, Pelletier KR, Murray JF, Sharda CE, Berger ML, Turpin RS, et al. Stanford presenteeism scale: health status and employee productivity. J Occup Environ Med. 2002;44(1):14-20. [DOI:10.1097/00043764-20020100000004] [PMID]

24. Brborović H, Brborović O, Brumen V, Pavleković G, Mustajbegović J. Are nurse presenteeism and patient safety culture associated: a cross-sectional study. Arch Industrial Hyg Toxicol. 2014;65(2):149-56. [DOI:10.2478/10004-1254-65-2014-2462] [PMID]

25. Shikiar R, Halpern MT, Rentz AM, Khan ZM. Development of the Health and Work Questionnaire (HWQ): an instrument for assessing workplace productivity in relation to worker health. Work. 2004;22(3):219-29. [DOI:10.1037/t56069-000]

26. Sutin AR, Stephan Y, Luchetti M, Aschwanden D, Strickhouser JE, Lee JH. et al. BMI, Weight Discrimination, and the Trajectory of Distress and WellBeing Across the Coronavirus Pandemic. Obesity. 2021;29(1):38-45. [DOI:10.1002/oby.23048] [PMID]

27. Field AE, Coakley EH, Must A, Spadano JL, Laird N, Dietz WH, et al. Impact of overweight on the risk of developing common chronic diseases during a 10-year period. Arch Intern Med. 2001;161(13):1581-6. [DOI:10.1001/archinte.161.13.1581] [PMID]
28. Saptavani A, Suwandi T, Wibowo A. Correlation between Body Mass Index on Presenteeism and Absenteeism on Dislipidemia Worker. Indian J Public Health Res Dev.2018; 9(1). [DOI:10.5958/09765506.2018.00001.3]

29. Sanchez Bustillos A, Vargas KG, Gomero-Cuadra R. Work productivity among adults with varied Body Mass Index: Results from a Canadian population-based survey. J Epidemiol Glob Health. 2015;5(2):191-9. [DOI:10.1016/j.jegh.2014.08.001] [PMID] [PMCID]

30. Kirkham HS, Clark BL, Bolas CA, Lewis GH, Jackson AS, Fisher D, et al. Which modifiable health risks are associated with changes in productivity costs? Popul Health Manag. 2015;18(1):30-8. [DOI:10.1089/pop.2014.0033] [PMID]

31. Webster RK, Liu R, Karimullina K, Hall I, Amlôt R, Rubin GJ. A systematic review of infectious illness Presenteeism: prevalence, reasons and risk factors. BMC Public Health. 2019;19(1):799. [DOI:10.1186/s12889019-7138-x] [PMID] [PMCID]

32. Allemann A, Siebenhüner K, Hämmig O. Predictors of Presenteeism Among Hospital Employees-A CrossSectional Questionnaire-Based Study in Switzerland. J Occup Environ Med. 2019;61(12):1004-10. [DOI:10.1097/JOM.0000000000001721] [PMID]

33. Aronsson G, Hagberg J, Björklund C, Aboagye E, Marklund S, Leineweber C, et al. Health and motivation as mediators of the effects of job demands, job control, job support, and role conflicts at work and home on sickness presenteeism and absenteeism. Int Arch Occup Environ Health. 2021;94(3):409-18. [PMID] [PMCID] [DOI:10.1007/s00420-020-01591-w]

34. Côté K, Lauzier M, Stinglhamber F. The relationship between presenteeism and job satisfaction: A mediated moderation model using work engagement and perceived organizational support. Eur Manag J. 2021;39(2):270-8. [DOI:10.1016/i.emj.2020.09.001]

35. Hemp P. Presenteeism: at work-but out of it. Harv Bus Rev. 2004;82(10):49-58 